\title{
Local three-nucleon interaction from chiral effective field theory
}

\author{
P. Navráti ${ }^{*}$ \\ Lawrence Livermore National Laboratory, L-414, P.O. Box 808, Livermore, CA 94551, USA
}

(Dated: October 27, 2018)

\begin{abstract}
The three-nucleon (NNN) interaction derived within the chiral effective field theory at the nextto-next-to-leading order $\left(\mathrm{N}^{2} \mathrm{LO}\right)$ is regulated with a function depending on the magnitude of the momentum transfer. The regulated NNN interaction is then local in the coordinate space, which is advantages for some many-body techniques. Matrix elements of the local chiral NNN interaction are evaluated in a three-nucleon basis. Using the $a b$ initio no-core shell model (NCSM) the NNN matrix elements are employed in ${ }^{3} \mathrm{H}$ and ${ }^{4} \mathrm{He}$ bound-state calculations.
\end{abstract}

PACS numbers: 21.60.Cs, 21.30.-x, 21.30.Fe

\section{INTRODUCTION}

Interactions among nucleons are governed by quantum chromodynamics (QCD). In the low-energy regime relevant to nuclear structure, QCD is non-perturbative, and, therefore, hard to solve. Thus, theory has been forced to resort to models for the interaction, which have limited physical basis. New theoretical developments, however, allow us connect QCD with low-energy nuclear physics. The chiral effective field theory $(\chi \mathrm{EFT})$ [1] provides a promising bridge. Beginning with the pionic or the nucleon-pion system [2] one works consistently with systems of increasing nucleon number $3,4,4,5$. One makes use of spontaneous breaking of chiral symmetry to systematically expand the strong interaction in terms of a generic small momentum and takes the explicit breaking of chiral symmetry into account by expanding in the pion mass. Thereby, the NN interaction, the NNN interaction and also $\pi \mathrm{N}$ scattering are related to each other. At the same time, the pion mass dependence of the interaction is known, which will enable a connection to lattice QCD calculations in the future [6]. Nuclear interactions are non-perturbative, because diagrams with purely nucleonic intermediate states are enhanced [1]. Therefore, the chiral perturbation expansion is performed for the potential (note, however, the discussion in Refs. 7, 8, 9] that points out some potential inconsitencies of this approach). Solving the Schrödinger equation for this potential then automatically sums diagrams with purely nucleonic intermediate states to all orders. The $\chi \mathrm{EFT}$ predicts, along with the NN interaction at the leading order, an NNN interaction at the 3rd order (next-to-next-to-leading order or $\mathrm{N}^{2} \mathrm{LO}$ ) [1, 10, 11], and even an NNNN interaction at the 4th order $\left(\mathrm{N}^{3} \mathrm{LO}\right)$ [12]. The details of $\mathrm{QCD}$ dynamics are contained in parameters, low-energy constants (LECs), not fixed by the symmetry. These parameters can be constrained by experiment. At present, high-quality $\mathrm{NN}$ potentials have been determined at order $\mathrm{N}^{3} \mathrm{LO}[13$. A crucial feature of $\chi \mathrm{EFT}$ is the consistency between the $\mathrm{NN}$, NNN and NNNN parts. As a consequence, at $\mathrm{N}^{2} \mathrm{LO}$ and $\mathrm{N}^{3} \mathrm{LO}$, except for two LECs, assigned to two NNN diagrams, the potential is fully constrained by the parameters defining the NN interaction.

It is of great interest and also a challenge to apply the chiral interactions in nuclear structure and nuclear reaction calculations. In a recent work [14], the presently available $\mathrm{NN}$ potential at $\mathrm{N}^{3} \mathrm{LO}$ [13] and the NNN interaction at $\mathrm{N}^{2} \mathrm{LO}$ [10, 11] have been applied to the calculation of various properties of $s$ - and $p$-shell nuclei, using the ab initio no-core shell model (NCSM) [15, 16], up to now the only approach able to handle the nonlocal $\chi \mathrm{EFT}$ NN potentials for systems beyond $A=4$. In that study, a preferred choice of the two NNN LECs, $c_{D}$ and $c_{E}$, was found and the fundamental importance of the $\chi \mathrm{EFT}$ NNN interaction was demonstrated for reproducing the structure of mid- $p$-shell nuclei. In a subsequent study, the same Hamiltonian was used to calculate microscopically the photo-absorption cross section of ${ }^{4} \mathrm{He}$ [17.

The approach of Ref. [14] differs in two aspects from the first NCSM application of the $\chi$ EFT NN+NNN interactions in Ref. [18], which presents a detailed investigation of ${ }^{7} \mathrm{Li}$. First, a regulator depending on the momentum transfer in the NNN terms was introduced which results in a local $\chi$ EFT NNN interaction. Second, the ${ }^{4}$ He binding energy was not used exclusively as the second constraint on the $c_{D}$ and $c_{E}$ LECs.

A local NNN interaction is advantages for some few- and many-body approaches because it is simpler to use. At the same time, it is known that details of the NNN interaction are important for nuclear structure applications. For example, the Urbana IX [19] and the Tucson-Melbourne [20, 21, 22] NNN interactions perform differently in mid-p-shell nuclei [16, 23, 24] although their differences appear to be minor. In the Green's function Monte Carlo 
(GFMC) calculations with the AV18 NN potential 25], the best results for $p$-shell nuclei up to $A=10$ are found using the Illinois NNN interaction that augments the Urbana IX by a two-pion term from the Tucson-Melbourne NNN interaction and by three-pion terms that in the $\chi \mathrm{EFT}$ appear beyond the $\mathrm{N}^{3} \mathrm{LO}$ [26, 27]. Contrary to the Illinois NNN interaction, the $\chi \mathrm{EFT}$ NNN interaction features the above mentioned consistency with the accompanying NN interaction. Still, interestingly, we found that the nonlocal $\chi$ EFT NNN interaction used in Ref. [18] and the local $\chi$ EFT NNN interaction employed in Ref. [14] differ to some extent in their description of mid- $p$-shell nuclei with the latter giving results in a better agreement with experiment. Therefore, it is important to pay attention to the details of the NNN interaction and test different possibilities.

It is the purpose of this paper to elaborate on the details of the local $\chi \mathrm{EFT}$ NNN interaction used in Refs. [14, 17] and present its matrix elements in the three-nucleon basis. Technical details of dealing with NNN interactions were investigated in many papers [28, 29, 30, 31, 32, 33, 34]. A new feature in the present work is the use of $\chi$ EFT contact interactions and a focus on the application within the ab initio NCSM. In particular, we demonstrate the bindingenergy convergence of the three-nucleon and four-nucleon systems with the $\chi$ EFT NN+NNN interactions using the $a b$ initio NCSM. In Sect. [II the local $\chi$ EFT NNN interaction is discussed and compared to the nonlocal version of Ref. [11]. Its three-nucleon matrix elements are given term by term. In Sect. [III the ${ }^{3} \mathrm{H}$ and ${ }^{4} \mathrm{He}$ binding energy and radius calculation results using the $\mathrm{N}^{3} \mathrm{LO} \chi \mathrm{EFT} \mathrm{NN}$ interaction of Ref. 13] and the local $\chi \mathrm{EFT} \mathrm{NNN}$ interaction are given. Conclusions are drawn in Sect. IV.

\section{LOCAL $\chi$ EFT NNN INTERACTION AT N ${ }^{2}$ LO}

The NNN interaction appearing at the third order $\left(\mathrm{N}^{2} \mathrm{LO}\right)$ of the $\chi$ EFT comprises of three parts: (i) The two-pion exchange, (ii) the one-pion exchange plus contact and the three-nucleon contact. In this section, we discuss all the

parts in detail and present the three-nucleon matrix elements of all the terms. For the two parts that contain the contact interactions, we also discuss in detail the impact of different regularization schemes.

\section{A. Three-nucleon coordinates}

We use the following definitions of the Jacobi coordinates

$$
\begin{aligned}
& \vec{\xi}_{1}=\frac{1}{\sqrt{2}}\left(\vec{r}_{1}-\vec{r}_{2}\right), \\
& \vec{\xi}_{2}=\sqrt{\frac{2}{3}}\left(\frac{1}{2}\left(\vec{r}_{1}+\vec{r}_{2}\right)-\vec{r}_{3}\right),
\end{aligned}
$$

and associated momenta

$$
\begin{aligned}
& \vec{\pi}_{1}=\frac{1}{\sqrt{2}}\left(\vec{p}_{1}-\vec{p}_{2}\right), \\
& \vec{\pi}_{2}=\sqrt{\frac{2}{3}}\left(\frac{1}{2}\left(\vec{p}_{1}+\vec{p}_{2}\right)-\vec{p}_{3}\right) .
\end{aligned}
$$

We also define the momenta transferred by nucleon 2 and nucleon 3 :

$$
\begin{aligned}
& \vec{Q}=\vec{p}_{2}^{\prime}-\vec{p}_{2}=-\frac{1}{\sqrt{2}}\left(\vec{\pi}_{1}^{\prime}-\vec{\pi}_{1}\right)+\frac{1}{\sqrt{6}}\left(\vec{\pi}_{2}^{\prime}-\vec{\pi}_{2}\right), \\
& \vec{Q}^{\prime}=\vec{p}_{3}^{\prime}-\vec{p}_{3}=-\sqrt{\frac{2}{3}}\left(\vec{\pi}_{2}^{\prime}-\vec{\pi}_{2}\right),
\end{aligned}
$$

where the primed coordinates refer to the initial momentum and the unprimed to the final momentum of the nucleon.

\section{B. General structure of three-nucleon interaction and its matrix element}

The NNN interaction is symmetric under permutation of the three nucleon indexes. It can be written as a sum of three pieces related by particle permutations:

$$
W=W_{1}+W_{2}+W_{3}
$$


To obtain its matrix element in an antisymmetrized three-nucleon basis we need to consider just a single term, e.g. $W_{1}$. In this paper, we use the basis of harmonic oscillator (HO) wave functions. However, most of the expressions have general validity. Following notation of Ref. [35], a general matrix element can be written as

$$
\begin{aligned}
\left\langle N i J T|W| N^{\prime} i^{\prime} J T\right\rangle=3\left\langle N i J T\left|W_{1}\right| N^{\prime} i^{\prime} J T\right\rangle= & 3 \sum\langle n l s j t, \mathcal{N} \mathcal{L} \mathcal{J} \| N i J T\rangle\left\langle n^{\prime} l^{\prime} s^{\prime} j^{\prime} t^{\prime}, \mathcal{N}^{\prime} \mathcal{L}^{\prime} \mathcal{J}^{\prime} \| N^{\prime} i^{\prime} J T\right\rangle \\
& \times\left\langle(n l s j t, \mathcal{N} \mathcal{L} \mathcal{J}) J T\left|W_{1}\right|\left(n^{\prime} l^{\prime} s^{\prime} j^{\prime} t^{\prime}, \mathcal{N}^{\prime} \mathcal{L}^{\prime} \mathcal{J}^{\prime}\right) J T\right\rangle,
\end{aligned}
$$

where $|N i J T\rangle$ is an antisymmetrized three-nucleon state with $N=2 n+l+2 \mathcal{N}+\mathcal{L}, i$ an additional quantum number and $J$ and $T$ the total angular momentum and total isospin, respectively. The parity of the state is $(-1)^{N}$. The state $|(n l s j t, \mathcal{N} \mathcal{L} \mathcal{J}) J T\rangle$ is a product of the HO wave functions $\left\langle\vec{\xi}_{1} \mid n l\right\rangle$ and $\left\langle\vec{\xi}_{2} \mid \mathcal{N} \mathcal{L}\right\rangle$ associated with the coordinates (1) and (2), respectively. This state is antisymmetrized only with respect to the exchange of nucleons 1 and 2 , i.e. $(-1)^{l+s+t}=-1$. The coefficient of fractional parentage $\langle n l s j t, \mathcal{N} \mathcal{L} \mathcal{J} \| N i J T\rangle$ is calculated according to Ref. 35].

\section{C. $\mathbf{N}^{2} \mathrm{LO}$ three-nucleon interaction contact term}

We start our discussion with the most trivial part of the $\chi \mathrm{EFT} \mathrm{N}^{2} \mathrm{LO}$ NNN interaction, the three-nucleon contact term

$$
W_{1}^{\text {cont }}=E \vec{\tau}_{2} \cdot \vec{\tau}_{3} \delta\left(\vec{r}_{1}-\vec{r}_{2}\right) \delta\left(\vec{r}_{3}-\vec{r}_{1}\right)=E \vec{\tau}_{2} \cdot \vec{\tau}_{3} \frac{1}{(2 \pi)^{6}} \frac{1}{(\sqrt{3})^{3}} \int \mathrm{d} \vec{\pi}_{1} \mathrm{~d} \vec{\pi}_{2} \mathrm{~d} \vec{\pi}_{1}^{\prime} \mathrm{d} \vec{\pi}_{2}^{\prime}\left|\vec{\pi}_{1} \vec{\pi}_{2}\right\rangle\left\langle\vec{\pi}_{1}^{\prime} \vec{\pi}_{2}^{\prime}\right|,
$$

with $E=\frac{c_{E}}{F_{\pi}^{4} \Lambda_{\chi}}$ where $\Lambda_{\chi}$ is the chiral symmetry breaking scale of the order of the $\rho$ meson mass and $F_{\pi}=92.4 \mathrm{MeV}$ is the weak pion decay constant. The $c_{E}$ is a low-energy constant (LEC) from the chiral Lagrangian of order one. The corresponding diagram is shown in Fig. 1. This term was regulated in Ref. [11] by a regulator dependent on the
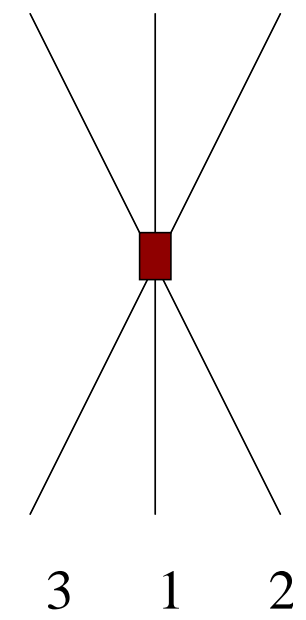

FIG. 1: Contact interaction NNN term of the $\mathrm{N}^{2} \mathrm{LO} \chi \mathrm{EFT}$.

sum of Jacobi momenta squared:

$$
W_{1}^{\text {cont,ENGKMW }}=E \vec{\tau}_{2} \cdot \vec{\tau}_{3} \frac{1}{(2 \pi)^{6}} \frac{1}{(\sqrt{3})^{3}} \int \mathrm{d} \vec{\pi}_{1} \mathrm{~d} \vec{\pi}_{2} \mathrm{~d} \vec{\pi}_{1}^{\prime} \mathrm{d} \vec{\pi}_{2}^{\prime}\left|\vec{\pi}_{1} \vec{\pi}_{2}\right\rangle F\left(\frac{1}{2}\left(\pi_{1}^{2}+\pi_{2}^{2}\right) ; \Lambda\right) F\left(\frac{1}{2}\left(\pi_{1}^{\prime 2}+\pi_{2}^{\prime 2}\right) ; \Lambda\right)\left\langle\vec{\pi}_{1}^{\prime} \vec{\pi}_{2}^{\prime}\right|,
$$

with the regulator function

$$
F\left(q^{2} ; \Lambda\right)=\exp \left(-q^{4} / \Lambda^{4}\right)
$$

with the limit $F\left(q^{2} ; \Lambda \rightarrow \infty\right)=1$. This was in particular convenient as the calculations were performed in momentum space.

Alternatively, let us consider a regulator dependent on momentum transfer:

$$
\begin{aligned}
W_{1}^{\text {cont }, \mathrm{Q}} & =E \vec{\tau}_{2} \cdot \vec{\tau}_{3} \frac{1}{(2 \pi)^{6}} \frac{1}{(\sqrt{3})^{3}} \int \mathrm{d} \vec{\pi}_{1} \mathrm{~d} \vec{\pi}_{2} \mathrm{~d} \vec{\pi}_{1}^{\prime} \mathrm{d} \vec{\pi}_{2}^{\prime}\left|\vec{\pi}_{1} \vec{\pi}_{2}\right\rangle F\left(\vec{Q}^{2} ; \Lambda\right) F\left(\vec{Q}^{\prime 2} ; \Lambda\right)\left\langle\vec{\pi}_{1}^{\prime} \vec{\pi}_{2}^{\prime}\right| \\
& =E \vec{\tau}_{2} \cdot \vec{\tau}_{3} \int \mathrm{d} \vec{\xi}_{1} \mathrm{~d} \vec{\xi}_{2}\left|\vec{\xi}_{1} \vec{\xi}_{2}\right\rangle Z_{0}\left(\sqrt{2} \xi_{1} ; \Lambda\right) Z_{0}\left(\left|\frac{1}{\sqrt{2}} \vec{\xi}_{1}+\sqrt{\frac{3}{2}} \vec{\xi}_{2}\right| ; \Lambda\right)\left\langle\vec{\xi}_{1} \vec{\xi}_{2}\right|
\end{aligned}
$$


where we introduced the function

$$
Z_{0}(r ; \Lambda)=\frac{1}{2 \pi^{2}} \int \mathrm{d} q q^{2} j_{0}(q r) F\left(q^{2} ; \Lambda\right) .
$$

This results in an interaction local in coordinate space because of the dependence of the regulator function on differences of initial and final Jacobi momenta. An interaction local in coordinate space may be more convenient for some methods. In fact, most of the NNN interactions used in few-body calculations, such as the Tucson-Melbourne $\left(\mathrm{TM}^{\prime}\right)$ [21, 22], Urbana IX (UIX) [19] or Illinois 2 (IL2) [26], are local in coordinate space.

The two alternatively regulated contact interactions lead to different three-nucleon matrix elements. The interaction (10) gives

$$
\begin{aligned}
& \left\langle(n l s j t, \mathcal{N} \mathcal{L} \mathcal{J}) J T\left|W_{1}^{\text {cont,ENGKMW }}\right|\left(n^{\prime} l^{\prime} s^{\prime} j^{\prime} t^{\prime}, \mathcal{N}^{\prime} \mathcal{L}^{\prime} \mathcal{J}^{\prime}\right) J T\right\rangle \\
= & E \frac{1}{2 \sqrt{3} \pi^{4}} \delta_{l 0} \delta_{\mathcal{L} 0} \delta_{l^{\prime} 0} \delta_{\mathcal{L}^{\prime} 0} \delta_{s s^{\prime}} \delta_{s j} \delta_{s^{\prime} j^{\prime}} \delta_{\mathcal{J} \frac{1}{2}} \delta_{\mathcal{J}^{\prime} \frac{1}{2}} \hat{t} \hat{t}^{\prime}(-1)^{t+t^{\prime}+T+\frac{1}{2}}\left\{\begin{array}{ccc}
t & t^{\prime} & 1 \\
\frac{1}{2} & \frac{1}{2} & \frac{1}{2}
\end{array}\right\}\left\{\begin{array}{ccc}
t & t^{\prime} & 1 \\
\frac{1}{2} & \frac{1}{2} & T
\end{array}\right\} \\
& \times \int \mathrm{d} \pi_{1} \mathrm{~d} \pi_{2} \pi_{1}^{2} \pi_{2}^{2}(-1)^{(n+\mathcal{N})} R_{n 0}\left(\pi_{1}, \frac{1}{b}\right) R_{\mathcal{N} 0}\left(\pi_{2}, \frac{1}{b}\right) F\left(\frac{1}{2}\left(\pi_{1}^{2}+\pi_{2}^{2}\right) ; \Lambda\right) \\
& \times \int \mathrm{d} \pi_{1}^{\prime} \mathrm{d} \pi_{2}^{\prime} \pi_{1}^{\prime 2} \pi_{2}^{\prime 2}(-1)^{\left(n^{\prime}+\mathcal{N}^{\prime}\right)} R_{n^{\prime} 0}\left(\pi_{1}^{\prime}, \frac{1}{b}\right) R_{\mathcal{N}^{\prime} 0}\left(\pi_{2}^{\prime}, \frac{1}{b}\right) F\left(\frac{1}{2}\left(\pi_{1}^{\prime 2}+\pi_{2}^{\prime 2}\right) ; \Lambda\right)
\end{aligned}
$$

while the interaction (12) results in the following matrix element:

$$
\begin{aligned}
& \left\langle(n l s j t, \mathcal{N} \mathcal{L} \mathcal{J}) J T\left|W_{1}^{\text {cont, }}\right|\left(n^{\prime} l^{\prime} s^{\prime} j^{\prime} t^{\prime}, \mathcal{N}^{\prime} \mathcal{L}^{\prime} \mathcal{J}^{\prime}\right) J T\right\rangle
\end{aligned}
$$

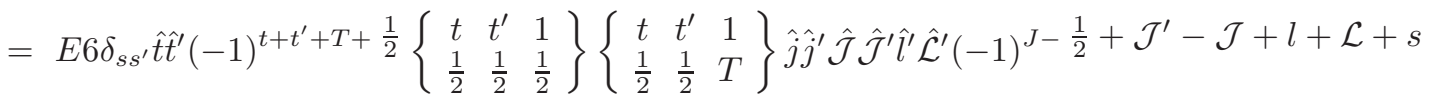

$$
\begin{aligned}
& \times \sum_{X}(-1)^{X} \hat{X}^{2}\left\{\begin{array}{ccc}
l^{\prime} & l & X \\
j & j^{\prime} & s
\end{array}\right\}\left\{\begin{array}{ccc}
j & j^{\prime} & X \\
\mathcal{J}^{\prime} & \mathcal{J} & J
\end{array}\right\}\left\{\begin{array}{ccc}
\mathcal{J}^{\prime} & \mathcal{J} & X \\
\mathcal{L} & \mathcal{L}^{\prime} & \frac{1}{2}
\end{array}\right\}\left(l^{\prime} 0 X 0 \mid l 0\right)\left(\mathcal{L}^{\prime} 0 X 0 \mid \mathcal{L} 0\right) \\
& \times \int \mathrm{d} \xi_{1} \mathrm{~d} \xi_{2} \xi_{1}^{2} \xi_{2}^{2} R_{n l}\left(\xi_{1}, b\right) R_{\mathcal{N} \mathcal{L}}\left(\xi_{2}, b\right) R_{n^{\prime} l^{\prime}}\left(\xi_{1}, b\right) R_{\mathcal{N}^{\prime} \mathcal{L}^{\prime}}\left(\xi_{2}, b\right) Z_{0}\left(\sqrt{2} \xi_{1} ; \Lambda\right) Z_{0, X}\left(\sqrt{\frac{1}{2}} \xi_{1}, \sqrt{\frac{3}{2}} \xi_{2} ; \Lambda\right) .
\end{aligned}
$$

In the above expressions, we have introduced the radial HO wave functions $R_{n l}$ with the oscillator parameter $b$ and, further, a new function

$$
Z_{0, X}\left(r_{1}, r_{2} ; \Lambda\right)=\frac{1}{2 \pi^{2}} \int \mathrm{d} q q^{2} j_{X}\left(q r_{1}\right) j_{X}\left(q r_{2}\right) F\left(q^{2} ; \Lambda\right)
$$

We also introduced the customary abriviation $\hat{l}=\sqrt{2 l+1}$. It should be noted that the two differently regulated contact interaction have different tensorial structure. One would perhaps expect that matrix elements of a local interaction will be easier to calculate. This is not the case for the discussed contact interaction. From Eq. (14) we can see that the term (10) acts only in $S$-waves. On the other hand, the local interaction (12) acts in higher partial waves as well as seen from Eq. (15). We display this schematically in Fig. 2 by breaking the symmetry of the pure contact interaction diagram (Fig. 1) and showing the finite range of the momentum transfer regulated interaction. Using $F\left(q^{2} ; \Lambda \rightarrow \infty\right)=1, Z_{0}(r, \Lambda \rightarrow \infty)=\frac{1}{4 \pi r^{2}} \delta(r)$ and $Z_{0, X}\left(r_{1}, r_{2} ; \Lambda \rightarrow \infty\right)=\frac{1}{4 \pi r_{1}^{2}} \delta\left(r_{1}-r_{2}\right)$, it is straightforward to verify that in the limit $\Lambda \rightarrow \infty$ both expressions (14) and (15) lead to the same result. For completeness, let us note that in Ref. [11] a matrix element of $\vec{\tau}_{1} \cdot \vec{\tau}_{2}$ was calculated, i.e. $\left\langle\vec{\tau}_{1} \cdot \vec{\tau}_{2}\right\rangle=6 \delta_{t t^{\prime}}(-1)^{t-1}\left\{\begin{array}{ccc}\frac{1}{2} & \frac{1}{2} t \\ \frac{1}{2} & \frac{1}{2} & 1\end{array}\right\}$, instead of $\vec{\tau}_{2} \cdot \vec{\tau}_{3}$ as we do in Eq. (14). Either choice lead to identical matrix element in the three-nucleon antisymmetrized basis (8). This is not the case once we regulate with the momentum transfer. Our choice in (12) results in the same isospin-coordinate structure as that obtained in Ref. [10].

\section{Transformation of the momentum part of the NNN interaction}

A general NNN interaction term is a product of isospin, spin and momentum parts. In this subsection, we manipulate the momentum part. We only consider the case of the regulator function $F\left(q^{2}, \Lambda\right)$ depending on transfered momentum. The momentum part of a general term $W_{1}$ can be schematically written as

$$
g_{K_{1}}(|\vec{Q}| ; \Lambda) g_{K_{2}}\left(\left|\vec{Q}^{\prime}\right| ; \Lambda\right)\left(Y_{K_{1}}(\hat{Q}) Y_{K_{2}}\left(\hat{Q}^{\prime}\right)\right)^{(K)},
$$




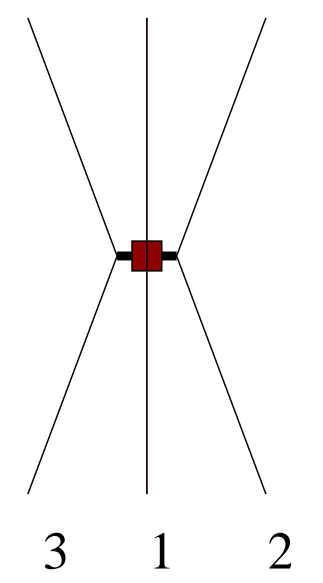

FIG. 2: Contact interaction NNN term of the $\mathrm{N}^{2} \mathrm{LO} \chi \mathrm{EFT}$ regulated by a function depending on momentum transfer.

with $K_{1}+K_{2}$ even and with $\vec{Q}$ and $\vec{Q}^{\prime}$ defined by Eqs. (3) and (4), respectively. For coordinates and momenta, $\hat{Q}$ denotes the angular part of the vector $\vec{Q}$. A transformation of (17) to coordinate space leads to a local interaction

$$
\begin{aligned}
& \frac{1}{(2 \pi)^{6}} \frac{1}{(\sqrt{3})^{3}} \int \mathrm{d} \vec{\pi}_{1} \mathrm{~d} \vec{\pi}_{2} \mathrm{~d} \vec{\pi}_{1}^{\prime} \mathrm{d} \vec{\pi}_{2}^{\prime}\left|\vec{\pi}_{1} \vec{\pi}_{2}\right\rangle g_{K_{1}}(|\vec{Q}| ; \Lambda) g_{K_{2}}\left(\left|\vec{Q}^{\prime}\right| ; \Lambda\right)\left(Y_{K_{1}}(\hat{Q}) Y_{K_{2}}\left(\hat{Q}^{\prime}\right)\right)^{(K)}\left\langle\vec{\pi}_{1}^{\prime} \vec{\pi}_{2}^{\prime}\right| \\
= & i^{K_{1}+K_{2}} \int \mathrm{d} \vec{\xi}_{1} \mathrm{~d} \vec{\xi}_{2}\left|\vec{\xi}_{1} \vec{\xi}_{2}\right\rangle f_{K_{1}}\left(\sqrt{2} \xi_{1} ; \Lambda\right) f_{K_{2}}\left(\left|\frac{1}{\sqrt{2}} \vec{\xi}_{1}+\sqrt{\frac{3}{2}} \vec{\xi}_{2}\right| ; \Lambda\right)\left(Y_{K_{1}}\left(\hat{\xi}_{1}\right) Y_{K_{2}}\left(\frac{1}{\sqrt{2}} \overrightarrow{\xi_{1}+\sqrt{\frac{3}{2}}} \vec{\xi}_{2}\right)\right)^{(K)}\left\langle\vec{\xi}_{1} \vec{\xi}_{2}\right| .
\end{aligned}
$$

Using (1) and (2), we note that $\sqrt{2} \vec{\xi}_{1}=\vec{r}_{1}-\vec{r}_{2}$ and $\frac{1}{\sqrt{2}} \vec{\xi}_{1}+\sqrt{\frac{3}{2}} \vec{\xi}_{2}=\vec{r}_{1}-\vec{r}_{3}$. In the above equation, we have introduced a new function using the relation

$$
i^{K} f_{K}(r ; \Lambda) Y_{K k}(\hat{r})=\frac{1}{(2 \pi)^{3}} \int \mathrm{d} \vec{q} \mathrm{e}^{i \vec{q} \cdot \vec{r}} g_{K}(q ; \Lambda) Y_{K k}(\hat{q})
$$

which implies

$$
f_{K}(r ; \Lambda)=\frac{1}{2 \pi^{2}} \int \mathrm{d} q q^{2} j_{K}(q r) g_{K}(q ; \Lambda) .
$$

We manipulate Eq. (18) first by utilizing the spherical harmonics relation

$$
Y_{K_{2} k_{2}}\left(\widehat{\vec{r}_{1}+\vec{r}_{2}}\right)=\sum_{K_{3}=0}^{K_{2}} \frac{\sqrt{4 \pi}}{\hat{K}_{3}}\left[\left(\begin{array}{c}
2 K_{2}+1 \\
2 K_{3}
\end{array}\right)\right]^{\frac{1}{2}} r_{1}^{K_{3}} r_{2}^{K_{2}-K_{3}}\left|\vec{r}_{1}+\vec{r}_{2}\right|^{-K_{2}}\left(Y_{K_{3}}\left(\hat{r}_{1}\right) Y_{K_{2}-K_{3}}\left(\hat{r}_{2}\right)\right)_{k_{2}}^{K_{2}},
$$

and, second, by the following expansion involving the functions depending on $\left|\frac{1}{\sqrt{2}} \vec{\xi}_{1}+\sqrt{\frac{3}{2}} \vec{\xi}_{2}\right|$ :

$$
f_{K_{2}}\left(\left|\vec{r}_{1}+\vec{r}_{2}\right| ; \Lambda\right)\left|\vec{r}_{1}+\vec{r}_{2}\right|^{-K_{2}}=4 \pi \sum_{X M_{X}} f_{K_{2}, X}\left(r_{1}, r_{2} ; \Lambda\right)(-1)^{X} Y_{X M_{X}}^{*}\left(\hat{r}_{1}\right) Y_{X M_{X}}\left(\hat{r}_{2}\right),
$$

with the function $f_{K_{2}, X}\left(r_{1}, r_{2} ; \Lambda\right)$ given by

$$
f_{K_{2}, X}\left(r_{1}, r_{2} ; \Lambda\right)=\frac{2}{\pi} \int \mathrm{d} q q^{2} j_{X}\left(q r_{1}\right) j_{X}\left(q r_{2}\right) \int \mathrm{d} r r^{2} j_{0}(q r) \frac{f_{K_{2}}(r ; \Lambda)}{r^{K_{2}}},
$$

or, equivalently, by

$$
f_{K_{2}, X}\left(r_{1}, r_{2} ; \Lambda\right)=\frac{1}{2} \int_{-1}^{1} \mathrm{~d} u P_{X}(u) \frac{f_{K_{2}}\left(\sqrt{r_{1}^{2}+r_{2}^{2}-2 r_{1} r_{2} u} ; \Lambda\right)}{\left(r_{1}^{2}+r_{2}^{2}-2 r_{1} r_{2} u\right)^{K_{2}}}
$$


Using (21) and (22), the term (18) is re-written in the form

$$
\begin{aligned}
= & i^{K_{1}+K_{2}} \int \mathrm{d} \vec{\xi}_{1} \mathrm{~d} \vec{\xi}_{2}\left|\vec{\xi}_{1} \vec{\xi}_{2}\right\rangle f_{K_{1}}\left(\sqrt{2} \xi_{1} ; \Lambda\right) \sum_{K_{3}=0 X Y Z V}^{K_{2}} \sum_{X}\left[\left(\begin{array}{cc}
2 K_{2}+1 \\
2 K_{3}
\end{array}\right)\right]^{\frac{1}{2}}\left(\frac{1}{\sqrt{2}} \xi_{1}\right)^{K_{3}}\left(\sqrt{\frac{3}{2}} \xi_{2}\right)^{K_{2}-K_{3}} f_{K_{2}, X}\left(\frac{1}{\sqrt{2}} \xi_{1}, \sqrt{\frac{3}{2}} \xi_{2} ; \Lambda\right) \\
& \times \hat{X}^{2} \widehat{K_{2}-K_{3}} \hat{K}_{1} \hat{K}_{2} \hat{Y}(-1)^{K_{1}+Y+Z+K}\left\{\begin{array}{ccc}
K_{1} & Y & V \\
Z & K & K_{2}
\end{array}\right\}\left\{\begin{array}{ccc}
Y & X & K_{3} \\
K_{2}-K_{3} & K_{2} & Z
\end{array}\right\} \\
& \times\left(X 0 K_{3} 0 \mid Y 0\right)\left(X 0 K_{2}-K_{3} 0 \mid Z 0\right)\left(Y 0 K_{1} 0 \mid V 0\right)\left(Y_{V}\left(\hat{\xi}_{1}\right) Y_{Z}\left(\hat{\xi}_{2}\right)\right)_{k}^{(K)}\left\langle\vec{\xi}_{1} \vec{\xi}_{2}\right|
\end{aligned}
$$

which is convenient for matrix element calculations.

\section{E. One-pion-exchange plus contact $\mathrm{N}^{2} \mathrm{LO}$ NNN term}

We are now in a position to discuss the one-pion exchange plus contact term that appears at the $\mathrm{N}^{2} \mathrm{LO}$. Following Ref. [1], we can write the $W_{1}$ term contribution as

$$
W_{1}^{1 \pi \_ \text {cont }}=-D \frac{1}{(2 \pi)^{6}} \frac{g_{\mathrm{A}}}{8 F_{\pi}^{2}} \vec{\tau}_{2} \cdot \vec{\tau}_{3}\left[\frac{1}{\vec{Q}^{\prime 2}+M_{\pi}^{2}} \vec{\sigma}_{2} \cdot \vec{Q}^{\prime} \vec{\sigma}_{3} \cdot \vec{Q}^{\prime}+\frac{1}{\vec{Q}^{2}+M_{\pi}^{2}} \vec{\sigma}_{2} \cdot \vec{Q} \vec{\sigma}_{3} \cdot \vec{Q}\right],
$$

with $D=\frac{c_{D}}{F_{\pi}^{2} \Lambda_{\chi}}$, where $c_{D}$ is a LEC from the chiral Lagrangian of order one. A diagramatic depiction of the second term in the parenthesis is presented in Fig. 3. The first term corresponds to the exchange of $2 \leftrightarrow 3$. Using the

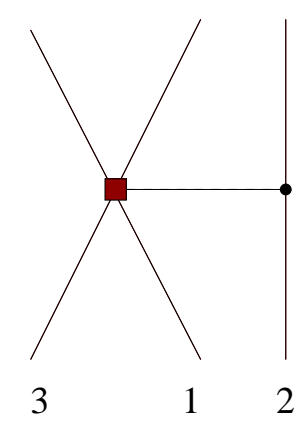

FIG. 3: One-pion exchage plus contact NNN interaction term of the $\mathrm{N}^{2} \mathrm{LO} \chi \mathrm{EFT}$.

regulator dependent on the sum of Jacobi momenta squared of Ref. [11], this term can be cast in the form

$$
\begin{aligned}
W_{1}^{1 \pi_{-} \text {cont,ENGKMW }}= & -D \frac{1}{(2 \pi)^{6}} \frac{g_{\mathrm{A}}}{8 F_{\pi}^{2}} \frac{1}{(\sqrt{3})^{3}} \int \mathrm{d} \vec{\pi}_{1} \mathrm{~d} \vec{\pi}_{2} \mathrm{~d} \vec{\pi}_{1}^{\prime} \mathrm{d} \vec{\pi}_{2}^{\prime}\left|\vec{\pi}_{1} \vec{\pi}_{2}\right\rangle F\left(\frac{1}{2}\left(\pi_{1}^{2}+\pi_{2}^{2}\right) ; \Lambda\right) \\
& \times \vec{\tau}_{2} \cdot \vec{\tau}_{3}\left[\frac{1}{\vec{Q}^{\prime 2}+M_{\pi}^{2}} \vec{\sigma}_{2} \cdot \vec{Q}^{\prime} \vec{\sigma}_{3} \cdot \vec{Q}^{\prime}+\frac{1}{\vec{Q}^{2}+M_{\pi}^{2}} \vec{\sigma}_{2} \cdot \vec{Q} \vec{\sigma}_{3} \cdot \vec{Q}\right] F\left(\frac{1}{2}\left(\pi_{1}^{\prime 2}+\pi_{2}^{\prime 2}\right) ; \Lambda\right)\left\langle\vec{\pi}_{1}^{\prime} \vec{\pi}_{2}^{\prime}\right| .
\end{aligned}
$$

On the other hand, with a regulator dependent on momentum transfer, we get

$$
\begin{aligned}
W_{1}^{1 \pi_{-} \text {cont }, \mathrm{Q}}= & -D \frac{1}{(2 \pi)^{6}} \frac{g_{\mathrm{A}}}{8 F_{\pi}^{2}} \frac{1}{(\sqrt{3})^{3}} \int \mathrm{d} \vec{\pi}_{1} \mathrm{~d} \vec{\pi}_{2} \mathrm{~d} \vec{\pi}_{1}^{\prime} \mathrm{d} \vec{\pi}_{2}^{\prime}\left|\vec{\pi}_{1} \vec{\pi}_{2}\right\rangle F\left(\vec{Q}^{2} ; \Lambda\right) \\
& \times \vec{\tau}_{2} \cdot \vec{\tau}_{3}\left[\frac{1}{\vec{Q}^{\prime 2}+M_{\pi}^{2}} \vec{\sigma}_{2} \cdot \vec{Q}^{\prime} \vec{\sigma}_{3} \cdot \vec{Q}^{\prime}+\frac{1}{\vec{Q}^{2}+M_{\pi}^{2}} \vec{\sigma}_{2} \cdot \vec{Q} \vec{\sigma}_{3} \cdot \vec{Q}\right] F\left(\vec{Q}^{\prime 2} ; \Lambda\right)\left\langle\vec{\pi}_{1}^{\prime} \vec{\pi}_{2}^{\prime}\right|,
\end{aligned}
$$

which leads to a term local in coordinate space. We depict the second term in the parenthesis of (28) schematically in Fig. 4 . The first term corresponds to the exchange of $2 \leftrightarrow 3$. This choice of regulation results in spin-isopincoordinate structure that also appears in NNN terms obtained in Ref. [10]. We note that a somewhat different spin-isopin structure was used for pion-range-short-range NNN terms in Refs. [32] and [34]. In Ref. [32] in particular, 
the $\vec{\sigma}$ and $\vec{\tau}$ operators were associated with the active nucleon 1, i.e.

$$
\begin{aligned}
W_{1}^{1 \pi_{-} \text {cont }, Q_{\sigma_{1}}=} & -D \frac{1}{(2 \pi)^{6}} \frac{g_{\mathrm{A}}}{8 F_{\pi}^{2}} \frac{1}{(\sqrt{3})^{3}} \int \mathrm{d} \vec{\pi}_{1} \mathrm{~d} \vec{\pi}_{2} \mathrm{~d} \vec{\pi}_{1}^{\prime} \mathrm{d} \vec{\pi}_{2}^{\prime}\left|\vec{\pi}_{1} \vec{\pi}_{2}\right\rangle F\left(\vec{Q}^{2} ; \Lambda\right) \\
& \times\left[\vec{\tau}_{1} \cdot \vec{\tau}_{3} \frac{1}{\vec{Q}^{\prime 2}+M_{\pi}^{2}} \vec{\sigma}_{1} \cdot \vec{Q}^{\prime} \vec{\sigma}_{3} \cdot \vec{Q}^{\prime}+\vec{\tau}_{1} \cdot \vec{\tau}_{2} \frac{1}{\vec{Q}^{2}+M_{\pi}^{2}} \vec{\sigma}_{1} \cdot \vec{Q} \vec{\sigma}_{2} \cdot \vec{Q}\right] F\left(\vec{Q}^{\prime 2} ; \Lambda\right)\left\langle\vec{\pi}_{1}^{\prime} \vec{\pi}_{2}^{\prime}\right|,
\end{aligned}
$$

This change does not alter the matrix element of (27) in the antisymmetrized three-nucleon basis. It will lead to a difference in the matrix element of (28). However, the dependence on the regulator is a higher order effect than the $\chi \mathrm{EFT}$ expansion order used to derive the NNN interaction. Therefore, these differences should have only minor overall effect. In fact, we confirmed in nuclear structure calculations such as those described in Ref. 14 that impact of the choice (27) or (28) is small in particular when the natural LECs values are used $\left(\left|c_{D}\right| \approx 1\right)$. However, a more significant impact of the choice of the regulator in particular on spin-orbit force sensitive observables is observed in the case of the two-pion-exchange terms as discussed in the Introduction.

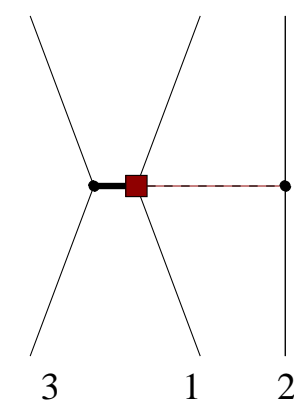

FIG. 4: One-pion exchage plus contact NNN interaction term of the $\mathrm{N}^{2} \mathrm{LO} \chi \mathrm{EFT}$ regulated by a function depending on momentum transfer.

Due to the antisymmetry of the three-nucleon wave functions in (8), it is sufficient to consider just one term of the two in parenthesis in (27) and (28) and multiply the result by two. Using the first term, the matrix element of (27) with the regulator dependent on the sum of Jacobi momenta squared is obtained in the form

$$
\begin{aligned}
& \left\langle(\text { nlsjt, } \mathcal{N} \mathcal{L} \mathcal{J}) J T\left|W_{1}^{1 \pi \text { cont,ENGKMW }}\right|\left(n^{\prime} l^{\prime} s^{\prime} j^{\prime} t^{\prime}, \mathcal{N}^{\prime} \mathcal{L}^{\prime} \mathcal{J}^{\prime}\right) J T\right\rangle \\
= & -D \frac{g_{\mathrm{A}}}{F_{\pi}^{2}} \frac{1}{2 \sqrt{3} \pi^{4}} \delta_{l 0} \delta_{l^{\prime} 0} \delta_{s j} \delta_{s^{\prime} j^{\prime}} \hat{t}^{\prime}\left\{\begin{array}{ccc}
t & t^{\prime} & 1 \\
\frac{1}{2} & \frac{1}{2} & \frac{1}{2}
\end{array}\right\}\left\{\begin{array}{ccc}
t & t^{\prime} & 1 \\
\frac{1}{2} & \frac{1}{2} & T
\end{array}\right\}(-1)^{\left(n+\mathcal{N}+n^{\prime}+\mathcal{N}^{\prime}\right)+\left(\mathcal{L}+\mathcal{L}^{\prime}\right) / 2} \\
& \times \hat{j} \hat{j}^{\prime} \hat{\mathcal{J}} \hat{\mathcal{J}}^{\prime}(-1)^{J-J}+T+\frac{1}{2}\left\{\begin{array}{ccc}
j & j^{\prime} & 1 \\
\frac{1}{2} & \frac{1}{2} & \frac{1}{2}
\end{array}\right\}\left\{\begin{array}{ccc}
j & j^{\prime} & 1 \\
\mathcal{J}^{\prime} & \mathcal{J} & J
\end{array}\right\} \sum_{K=0,2} \hat{K}(1010 \mid K 0)\left\{\begin{array}{ccc}
\mathcal{J} & \mathcal{L} & \frac{1}{2} \\
\mathcal{J}^{\prime} & \mathcal{L}^{\prime} & \frac{1}{2} \\
1 & K & 1
\end{array}\right\} \\
& \times \sum_{K_{1}=0}^{K} \widehat{K-K_{1}}\left[\left(\begin{array}{c}
2 K+1 \\
2 K_{1}
\end{array}\right)\right]^{\frac{1}{2}}(-1)^{\mathcal{L}+K_{1}} \sum_{X} \hat{X} \hat{\mathcal{L}}^{\prime}\left(K_{1} 0 X 0 \mid \mathcal{L} 0\right)\left(\mathcal{L}^{\prime} 0 K-K_{1} 0 \mid X 0\right)\left\{\begin{array}{ccc}
\mathcal{L}^{\prime} & K-K_{1} & X \\
K_{1} & \mathcal{L} & K
\end{array}\right\} \\
& \times \int \mathrm{d} \pi_{1} \mathrm{~d} \pi_{2} \mathrm{~d} \pi_{1}^{\prime} \mathrm{d} \pi_{2}^{\prime} \pi_{1}^{2} \pi_{2}^{2} \pi_{1}^{\prime 2} \pi_{2}^{\prime 2} R_{n 0}\left(\pi_{1}, \frac{1}{b}\right) R_{\mathcal{N} \mathcal{L}}\left(\pi_{2}, \frac{1}{b}\right) F\left(\frac{1}{2}\left(\pi_{1}^{2}+\pi_{2}^{2}\right) ; \Lambda\right) \\
& \times R_{n^{\prime} 0}\left(\pi_{1}^{\prime}, \frac{1}{b}\right) R_{\mathcal{N}^{\prime} \mathcal{L}^{\prime}}\left(\pi_{2}^{\prime}, \frac{1}{b}\right) F\left(\frac{1}{2}\left(\pi_{1}^{\prime 2}+\pi_{2}^{\prime 2}\right) ; \Lambda\right) \pi_{2}^{K_{1}} \pi_{2}^{\prime K-K_{1}} g_{K, X}\left(\pi_{2}, \pi_{2}^{\prime}\right),
\end{aligned}
$$

where we introduced the function

$$
g_{K, X}\left(p, p^{\prime}\right)=\frac{2}{\pi} \int \mathrm{d} q q^{2} d r r^{2} \frac{q^{2-K}}{\frac{2}{3} q^{2}+M_{\pi}^{2}} j_{0}(q r) j_{X}(p r) j_{X}\left(p^{\prime} r\right),
$$

which can be alternatively evaluated through

$$
g_{K, X}\left(p, p^{\prime}\right)=\frac{1}{2} \int_{-1}^{1} \mathrm{~d} u P_{X}(u) \frac{{\sqrt{p^{2}+p^{\prime 2}-2 p p^{\prime} u}}^{2-K}}{\frac{2}{3}\left(p^{2}+p^{\prime 2}-2 p p^{\prime} u\right)^{2}+M_{\pi}^{2}} .
$$

The matrix element (30) was first derived in Ref. [11]. 
For the one-pion exchange plus contact term (28) with the regulator dependent on momentum transfer, we present the matrix element obtained using both terms in the parenthesis of (28). Due to the three-nucleon wave function antisymmetry, both contributions lead to the same result for (8). One can take the advantage of this feature and use the alternative calculations to check the correctness of the numerical code. First, we take the first part of (28) and get

$$
\begin{aligned}
& \left\langle(n l s j t, \mathcal{N} \mathcal{L} \mathcal{J}) J T\left|W_{1}^{1 \pi \_ \text {cont }, Q}\right|\left(n^{\prime} l^{\prime} s^{\prime} j^{\prime} t^{\prime}, \mathcal{N}^{\prime} \mathcal{L}^{\prime} \mathcal{J}^{\prime}\right) J T\right\rangle \\
& =-D \frac{9 g_{\mathrm{A}}}{F_{\pi}^{2}} \hat{t} \hat{t}^{\prime}(-1)^{t+t^{\prime}+T+\frac{1}{2}}\left\{\begin{array}{ccc}
t & t^{\prime} & 1 \\
\frac{1}{2} & \frac{1}{2} & \frac{1}{2}
\end{array}\right\}\left\{\begin{array}{ccc}
t & t^{\prime} & 1 \\
\frac{1}{2} & \frac{1}{2} & T
\end{array}\right\} \hat{j} \hat{j}^{\prime} \hat{\mathcal{J}} \hat{\mathcal{J}}^{\prime}(-1)^{J-\mathcal{J}+s+j^{\prime}}\left\{\begin{array}{ccc}
s & s^{\prime} & 1 \\
\frac{1}{2} & \frac{1}{2} & \frac{1}{2}
\end{array}\right\} \\
& \times \sum_{K=0,2} \hat{K}(-1)^{(K / 2)}(1010 \mid K 0) \sum_{K_{2}, K_{3}} \hat{K}_{2} \hat{K}_{3} \hat{l}^{\prime} \hat{\mathcal{L}}^{\prime}\left(l^{\prime} 0 K_{2} 0 \mid l 0\right)\left(\mathcal{L}^{\prime} 0 K_{3} 0 \mid \mathcal{L} 0\right) \\
& \times \sum_{Z} \hat{Z}^{2}\left\{\begin{array}{ccc}
l & s & j \\
l^{\prime} & s^{\prime} & j^{\prime} \\
K_{2} & 1 & Z
\end{array}\right\}\left\{\begin{array}{ccc}
\mathcal{L} & \frac{1}{2} & \mathcal{J} \\
\mathcal{L}^{\prime} & \frac{1}{2} & \mathcal{J}^{\prime} \\
K_{3} & 1 & Z
\end{array}\right\}\left\{\begin{array}{ccc}
j & j^{\prime} & Z \\
\mathcal{J}^{\prime} & \mathcal{J} & J
\end{array}\right\}\left\{\begin{array}{ccc}
K_{2} & 1 & Z \\
1 & K_{3} & K
\end{array}\right\} \\
& \times \sum_{K_{1}=0}^{K} \widehat{K-K_{1}}\left[\left(\begin{array}{c}
2 K+1 \\
2 K_{1}
\end{array}\right)\right]^{\frac{1}{2}}(-1)^{K_{1}} \sum_{X}(-1)^{X} \hat{X}^{2}\left(K_{1} 0 X 0 \mid K_{2} 0\right)\left(K-K_{1} 0 X 0 \mid K_{3} 0\right)\left\{\begin{array}{llc}
K_{1} & X & K_{2} \\
K_{3} & K & K-K_{1}
\end{array}\right\} \\
& \times \int \mathrm{d} \xi_{1} \mathrm{~d} \xi_{2} \xi_{1}^{2} \xi_{2}^{2} R_{n l}\left(\xi_{1}, b\right) R_{\mathcal{N} \mathcal{L}}\left(\xi_{2}, b\right) R_{n^{\prime} l^{\prime}}\left(\xi_{1}, b\right) R_{\mathcal{N}^{\prime} \mathcal{L}^{\prime}}\left(\xi_{2}, b\right) \\
& \times\left(\sqrt{\frac{1}{2}} \xi_{1}\right)^{K_{1}}\left(\sqrt{\frac{3}{2}} \xi_{2}\right)^{K-K_{1}} Z_{0}\left(\sqrt{2} \xi_{1} ; \Lambda\right) f_{K, X}\left(\sqrt{\frac{1}{2}} \xi_{1}, \sqrt{\frac{3}{2}} \xi_{2} ; \Lambda\right),
\end{aligned}
$$

with the functions

$$
f_{0, X}\left(r_{1}, r_{2} ; \Lambda\right)=\frac{1}{2 \pi^{2}} \int \mathrm{d} q q^{2} j_{X}\left(q r_{1}\right) j_{X}\left(q r_{2}\right) \frac{q^{2} F\left(q^{2} ; \Lambda\right)}{q^{2}+M_{\pi}^{2}}
$$

and

$$
f_{2, X}\left(r_{1}, r_{2} ; \Lambda\right)=\frac{1}{4 \pi^{2}} \int_{0}^{\infty} \mathrm{d} q q^{2} j_{X}\left(q r_{1}\right) j_{X}\left(q r_{2}\right) \int_{q}^{\infty} \mathrm{d} k k\left(k^{2}-q^{2}\right) \frac{F\left(k^{2} ; \Lambda\right)}{k^{2}+M_{\pi}^{2}},
$$

which are special cases of (23). An alternative way of evaluating (35) is

$$
f_{2, X}\left(r_{1}, r_{2} ; \Lambda\right)=\frac{1}{2} \int_{-1}^{1} \mathrm{~d} u P_{X}(u) \frac{f_{2}\left(\sqrt{r_{1}^{2}+r_{2}^{2}-2 r_{1} r_{2} u} ; \Lambda\right)}{r_{1}^{2}+r_{2}^{2}-2 r_{1} r_{2} u},
$$

with

$$
f_{2}(r ; \Lambda)=\frac{1}{2 \pi^{2}} \int \mathrm{d} q q^{2} j_{2}(q r) \frac{q^{2} F\left(q^{2} ; \Lambda\right)}{q^{2}+M_{\pi}^{2}} .
$$

We note that (36) is numerically more efficient than (35).

Next, we take the second part of (28), which results in a simpler expression for one-pion-exchange plus contact $\mathrm{N}^{2} \mathrm{LO}$ three-nucleon matrix element in non-antisymmetrized basis:

$$
\begin{aligned}
& \left\langle(n l s j t, \mathcal{N} \mathcal{L} \mathcal{J}) J T\left|W_{1}^{1 \pi_{-} \text {cont, }}\right|\left(n^{\prime} l^{\prime} s^{\prime} j^{\prime} t^{\prime}, \mathcal{N}^{\prime} \mathcal{L}^{\prime} \mathcal{J}^{\prime}\right) J T\right\rangle \\
& =-D \frac{9 g_{\mathrm{A}}}{F_{\pi}^{2}} \hat{t} \hat{t}^{\prime}(-1)^{t+t^{\prime}+T+\frac{1}{2}}\left\{\begin{array}{ccc}
t & t^{\prime} & 1 \\
\frac{1}{2} & \frac{1}{2} & \frac{1}{2}
\end{array}\right\}\left\{\begin{array}{ccc}
t & t^{\prime} & 1 \\
\frac{1}{2} & \frac{1}{2} & T
\end{array}\right\} \hat{j} \hat{j}^{\prime} \hat{\mathcal{J}} \hat{\mathcal{J}}^{\prime} \hat{s} \hat{s}^{\prime}(-1)^{J-\mathcal{J}+s+j^{\prime}}\left\{\begin{array}{lll}
s & s^{\prime} & 1 \\
\frac{1}{2} & \frac{1}{2} & \frac{1}{2}
\end{array}\right\} \hat{l}^{\prime} \hat{\mathcal{L}}^{\prime} \\
& \times \sum_{K=0,2} \hat{K}(-1)^{(K / 2)}(1010 \mid K 0) \sum_{V}(-1)^{V} \hat{V}\left(V 0 l^{\prime} 0 \mid l 0\right) \sum_{X} \hat{X}^{2}(X 0 K 0 \mid V 0)\left(X 0 \mathcal{L}^{\prime} 0 \mid \mathcal{L} 0\right) \\
& \times \sum_{Z} \hat{Z}^{2}\left\{\begin{array}{ccc}
l & s & j \\
l^{\prime} & s^{\prime} & j^{\prime} \\
V & 1 & Z
\end{array}\right\}\left\{\begin{array}{ccc}
\mathcal{L} & \frac{1}{2} & \mathcal{J} \\
\mathcal{L}^{\prime} & \frac{1}{2} & \mathcal{J}^{\prime} \\
X & 1 & Z
\end{array}\right\}\left\{\begin{array}{ccc}
j & j^{\prime} & Z \\
\mathcal{J}^{\prime} & \mathcal{J} & J
\end{array}\right\}\left\{\begin{array}{ccc}
V & 1 & Z \\
1 & X & K
\end{array}\right\} \\
& \times \int \mathrm{d} \xi_{1} \mathrm{~d} \xi_{2} \xi_{1}^{2} \xi_{2}^{2} R_{n l}\left(\xi_{1}, b\right) R_{\mathcal{N} \mathcal{L}}\left(\xi_{2}, b\right) R_{n^{\prime} l^{\prime}}\left(\xi_{1}, b\right) R_{\mathcal{N}^{\prime} \mathcal{L}^{\prime}}\left(\xi_{2}, b\right) f_{K}\left(\sqrt{2} \xi_{1} ; \Lambda\right) Z_{0, X}\left(\sqrt{\frac{1}{2}} \xi_{1}, \sqrt{\frac{3}{2}} \xi_{2} ; \Lambda\right),
\end{aligned}
$$


with

$$
f_{K}(r ; \Lambda)=\frac{1}{2 \pi^{2}} \int \mathrm{d} q q^{2} j_{K}(q r) \frac{q^{2} F\left(q^{2} ; \Lambda\right)}{q^{2}+M_{\pi}^{2}},
$$

which is a special case of (20) and $Z_{0, X}\left(r_{1}, r_{2} ; \Lambda\right)$ given by Eq. (16). Both (33) and (38) are already multiplied by two in anticipation of the three-nucleon antisymmetry in the final matrix element (8).

For completeness, we also present the matrix element of the second part of (29):

$$
\begin{aligned}
& \left\langle(n l s j t, \mathcal{N} \mathcal{L} \mathcal{J}) J T\left|W_{1}^{1 \pi_{-} \text {cont, } Q_{\sigma_{1}}}\right|\left(n^{\prime} l^{\prime} s^{\prime} j^{\prime} t^{\prime}, \mathcal{N}^{\prime} \mathcal{L}^{\prime} \mathcal{J}^{\prime}\right) J T\right\rangle
\end{aligned}
$$

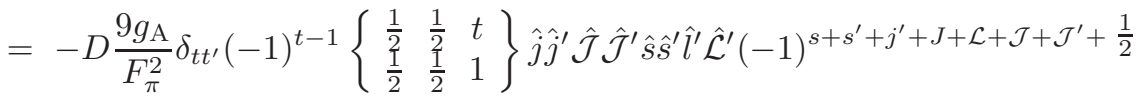

$$
\begin{aligned}
& \times \sum_{K=0,2} \hat{K}(-1)^{(K / 2)}(1010 \mid K 0)\left\{\begin{array}{ccc}
s & s^{\prime} & K \\
\frac{1}{2} & \frac{1}{2} & 1 \\
\frac{1}{2} & \frac{1}{2} & 1
\end{array}\right\} \sum_{V} \hat{V}\left(V 0 l^{\prime} 0 \mid l 0\right) \sum_{X} \hat{X}^{2}(X 0 K 0 \mid V 0)\left(X 0 \mathcal{L}^{\prime} 0 \mid \mathcal{L} 0\right) \\
& \times\left\{\begin{array}{ccc}
l & s & j \\
l^{\prime} & s^{\prime} & j^{\prime} \\
V & K & X
\end{array}\right\}\left\{\begin{array}{ccc}
\mathcal{J} & X & \mathcal{J}^{\prime} \\
j^{\prime} & J & j
\end{array}\right\}\left\{\begin{array}{ccc}
\mathcal{J} & X & \mathcal{J}^{\prime} \\
\mathcal{L}^{\prime} & \frac{1}{2} & \mathcal{L}
\end{array}\right\} \\
& \times \int \mathrm{d} \xi_{1} \mathrm{~d} \xi_{2} \xi_{1}^{2} \xi_{2}^{2} R_{n l}\left(\xi_{1}, b\right) R_{\mathcal{N} \mathcal{L}}\left(\xi_{2}, b\right) R_{n^{\prime} l^{\prime}}\left(\xi_{1}, b\right) R_{\mathcal{N}^{\prime} \mathcal{L}^{\prime}}\left(\xi_{2}, b\right) f_{K}\left(\sqrt{2} \xi_{1} ; \Lambda\right) Z_{0, X}\left(\sqrt{\frac{1}{2}} \xi_{1}, \sqrt{\frac{3}{2}} \xi_{2} ; \Lambda\right),
\end{aligned}
$$

which is still simpler than (38). Again, this matrix element is already multiplied by two in anticipation of the threenucleon antisymmetry in the final matrix element (8). The matrix element of the (two-times the) first part of (29) is given by (33) multiplied by $(-1)^{t+t^{\prime}+s+s^{\prime}}$. Due to the three-nucleon wave function antisymmetry, this contribution leads to the same result for (8) as does (38), which can be taken advantage of in testing the correctness of numerical calculations.

By comparing (27) with (33) (or equivalently with (38) and also with (40)) we note the different tensorial structure of the matrix elements. When the regulator dependent on the sum of Jacobi momenta squared is used, only the $l=0$, $l^{\prime}=0$ partial waves contribute. This is not the case, when the regulator depending on momentum transfer is utilized. At the same time, however, we note that in the limit $\Lambda \rightarrow \infty$ both expressions (27) and (33) as well as (38) and (40) lead to the same result.

\section{F. Two-pion exchange $\mathrm{N}^{2} \mathrm{LO}$ NNN terms}

In this subsection, we present matrix elements of two-pion exchange $\mathrm{N}^{2} \mathrm{LO}$ NNN terms. Their schematic depiction is shown in Fig. 囵. There are three distinct terms associated with three LECs, $c_{1}, c_{3}$ and $c_{4}$, from the chiral Lagrangian,

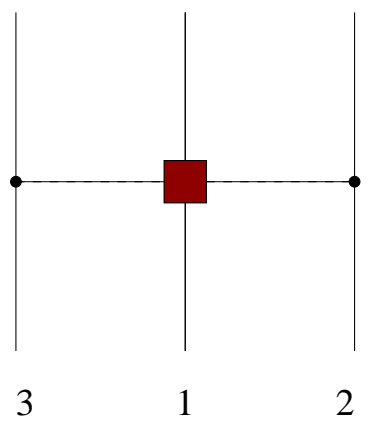

FIG. 5: Two-pion exchange NNN interaction term of the $\mathrm{N}^{2} \mathrm{LO} \chi \mathrm{EFT}$.

which also appear in the subleading two-pion exchange in the NN potential. Consequently, values of these LECs expected to be of order one are typically fixed at the NN level unlike the case of the previously introduced $c_{D}$ (26) and $c_{E}$ (9) LECs whose values needs to be fixed in systems of more than two nucleons. In the present paper, we derive only the matrix elements of the two-pion exchange NNN interaction terms regulated by a function depending on momentum transfer, i.e. terms that are local in coordinate space. 
Following Ref. [1], the $W_{1}$ part of the $c_{1}$ term with the momentum transfer regulators can be written as

$$
W_{1}^{2 \pi \_c 1}=-c_{1} \frac{1}{(2 \pi)^{6}} \frac{4 M_{\pi}^{2}}{F_{\pi}^{2}} \frac{g_{\mathrm{A}}^{2}}{4 F_{\pi}^{2}} \vec{\tau}_{2} \cdot \vec{\tau}_{3} F\left(\vec{Q}^{2} ; \Lambda\right) \frac{1}{\vec{Q}^{2}+M_{\pi}^{2}} \vec{\sigma}_{2} \cdot \vec{Q} \vec{\sigma}_{3} \cdot \vec{Q}^{\prime} \frac{1}{\vec{Q}^{\prime 2}+M_{\pi}^{2}} F\left(\vec{Q}^{\prime 2} ; \Lambda\right) .
$$

Using results of Subsect. IID we find for the $c_{1}$-term matrix element:

$$
\begin{aligned}
& \left\langle(n l s j t, \mathcal{N} \mathcal{L} \mathcal{J}) J T\left|W_{1}^{2 \pi \_c 1, Q}\right|\left(n^{\prime} l^{\prime} s^{\prime} j^{\prime} t^{\prime}, \mathcal{N}^{\prime} \mathcal{L}^{\prime} \mathcal{J}^{\prime}\right) J T\right\rangle \\
& =-c_{1} \frac{36 M_{\pi}^{2}}{F_{\pi}^{2}} \frac{g_{\mathrm{A}}^{2}}{F_{\pi}^{2}} \hat{t} \hat{t}^{\prime}(-1)^{t+t^{\prime}+T+\frac{1}{2}}\left\{\begin{array}{ccc}
t & t^{\prime} & 1 \\
\frac{1}{2} & \frac{1}{2} & \frac{1}{2}
\end{array}\right\}\left\{\begin{array}{ccc}
t & t^{\prime} & 1 \\
\frac{1}{2} & \frac{1}{2} & T
\end{array}\right\} \hat{j} \hat{j}^{\prime} \hat{\mathcal{J}} \hat{\mathcal{J}}^{\prime} \hat{s} \hat{s}^{\prime}(-1)^{J-\mathcal{J}+s+j^{\prime}}\left\{\begin{array}{ccc}
s & s^{\prime} & 1 \\
\frac{1}{2} & \frac{1}{2} & \frac{1}{2}
\end{array}\right\} \hat{l}^{\prime} \hat{\mathcal{L}}^{\prime} \\
& \times \sum_{V R} \hat{V} \hat{R}\left(V 0 l^{\prime} 0 \mid l 0\right)\left(R 0 \mathcal{L}^{\prime} 0 \mid \mathcal{L} 0\right) \sum_{Y}(-1)^{Y} \hat{Y}(Y 010 \mid V 0)\left\{\begin{array}{ccc}
l & s & j \\
l^{\prime} & s^{\prime} & j^{\prime} \\
V & 1 & Y
\end{array}\right\}\left\{\begin{array}{ccc}
\mathcal{L} & \frac{1}{2} & \mathcal{J} \\
\mathcal{L}^{\prime} & \frac{1}{2} & \mathcal{J}^{\prime} \\
R & 1 & Y
\end{array}\right\}\left\{\begin{array}{ccc}
j & j^{\prime} & Y \\
\mathcal{J}^{\prime} & \mathcal{J} & J
\end{array}\right\} \\
& \times \sum_{K_{3}=0}^{1}\left[\left(\begin{array}{c}
3 \\
2 K_{3}
\end{array}\right)\right]^{\frac{1}{2}} \widehat{1-K_{3}} \sum_{X} \hat{X}^{2}\left(X 0 K_{3} 0 \mid Y 0\right)\left(X 01-K_{3} 0 \mid R 0\right)\left\{\begin{array}{ccc}
Y & X & K_{3} \\
1-K_{3} & 1 & R
\end{array}\right\} \\
& \times \int \mathrm{d} \xi_{1} \mathrm{~d} \xi_{2} \xi_{1}^{2} \xi_{2}^{2} R_{n l}\left(\xi_{1}, b\right) R_{\mathcal{N} \mathcal{L}}\left(\xi_{2}, b\right) R_{n^{\prime} l^{\prime}}\left(\xi_{1}, b\right) R_{\mathcal{N}^{\prime} \mathcal{L}^{\prime}}\left(\xi_{2}, b\right) \\
& \times\left(\sqrt{\frac{1}{2}} \xi_{1}\right)^{K_{3}}\left(\sqrt{\frac{3}{2}} \xi_{2}\right)^{1-K_{3}} f_{1}\left(\sqrt{2} \xi_{1} ; \Lambda\right) f_{1, X}\left(\sqrt{\frac{1}{2}} \xi_{1}, \sqrt{\frac{3}{2}} \xi_{2} ; \Lambda\right) .
\end{aligned}
$$

Here we introduced the functions

$$
f_{1}(r ; \Lambda)=\frac{1}{2 \pi^{2}} \int \mathrm{d} q q^{2} j_{1}(q) \frac{q F\left(q^{2} ; \Lambda\right)}{q^{2}+M_{\pi}^{2}}
$$

and

$$
f_{1, X}\left(r_{1}, r_{2} ; \Lambda\right)=\frac{1}{2 \pi^{2}} \int_{0}^{\infty} \mathrm{d} q q^{2} j_{X}\left(q r_{1}\right) j_{X}\left(q r_{2}\right) \int_{q}^{\infty} \mathrm{d} k \frac{k F\left(k^{2} ; \Lambda\right)}{k^{2}+M_{\pi}^{2}},
$$

which are the explicit versions of functions given in Eqs. (20) and (23), respectively. The function (44) can be alternatively evaluated with the help of the Legendre polynomial:

$$
f_{1, X}\left(r_{1}, r_{2} ; \Lambda\right)=\frac{1}{2} \int_{-1}^{1} \mathrm{~d} u P_{X}(u) \frac{f_{1}\left(\sqrt{r_{1}^{2}+r_{2}^{2}-2 r_{1} r_{2} u} ; \Lambda\right)}{\sqrt{r_{1}^{2}+r_{2}^{2}-2 r_{1} r_{2} u}} .
$$

The $W_{1}$ part of the two-pion exchange $c_{3}$ term is given by [1]

$$
W_{1}^{2 \pi_{-} \mathrm{c} 3}=c_{3} \frac{1}{(2 \pi)^{6}} \frac{2}{F_{\pi}^{2}} \frac{g_{\mathrm{A}}^{2}}{4 F_{\pi}^{2}} \vec{\tau}_{2} \cdot \vec{\tau}_{3} F\left(\vec{Q}^{2} ; \Lambda\right) \frac{1}{\vec{Q}^{2}+M_{\pi}^{2}} \vec{\sigma}_{2} \cdot \vec{Q} \vec{\sigma}_{3} \cdot \vec{Q}^{\prime} \vec{Q} \cdot \vec{Q}^{\prime} \frac{1}{\vec{Q}^{\prime 2}+M_{\pi}^{2}} F\left(\vec{Q}^{\prime 2} ; \Lambda\right) .
$$

For its matrix element we find

$$
\begin{aligned}
& \left\langle(n l s j t, \mathcal{N} \mathcal{L} \mathcal{J}) J T\left|W_{1}^{2 \pi_{-} \mathrm{c} 3, \mathrm{Q}}\right|\left(n^{\prime} l^{\prime} s^{\prime} j^{\prime} t^{\prime}, \mathcal{N}^{\prime} \mathcal{L}^{\prime} \mathcal{J}^{\prime}\right) J T\right\rangle \\
& =c_{3} \frac{18}{F_{\pi}^{2}} \frac{g_{\mathrm{A}}^{2}}{F_{\pi}^{2}} \hat{t} \hat{t}^{\prime}(-1)^{t+t^{\prime}+T+\frac{1}{2}}\left\{\begin{array}{ccc}
t & t^{\prime} & 1 \\
\frac{1}{2} & \frac{1}{2} & \frac{1}{2}
\end{array}\right\}\left\{\begin{array}{ccc}
t & t^{\prime} & 1 \\
\frac{1}{2} & \frac{1}{2} & T
\end{array}\right\} \hat{j} \hat{j}^{\prime} \hat{\mathcal{J}} \hat{\mathcal{J}}^{\prime} \hat{s} \hat{s}^{\prime}(-1)^{J-\mathcal{J}+s+j^{\prime}}\left\{\begin{array}{lll}
s & s^{\prime} & 1 \\
\frac{1}{2} & \frac{1}{2} & \frac{1}{2}
\end{array}\right\} \hat{l}^{\prime} \hat{\mathcal{L}}^{\prime} \\
& \times \sum_{K_{1} K_{2}}(-1)^{\left(K_{1}+K_{2}\right) / 2} \hat{K}_{1} \hat{K}_{2}\left(1010 \mid K_{1} 0\right)\left(1010 \mid K_{2} 0\right) \\
& \times \sum_{V R}(-1)^{V+R} \hat{V} \hat{R}\left(V 0 l^{\prime} 0 \mid l 0\right)\left(R 0 \mathcal{L}^{\prime} 0 \mid \mathcal{L} 0\right) \sum_{Y} \hat{Y}\left(Y 0 K_{1} 0 \mid V 0\right) \\
& \times \sum_{Z} \hat{Z}^{2}(-1)^{Z}\left\{\begin{array}{ccc}
l & s & j \\
l^{\prime} & s^{\prime} & j^{\prime} \\
V & 1 & Z
\end{array}\right\}\left\{\begin{array}{ccc}
\mathcal{L} & \frac{1}{2} & \mathcal{J} \\
\mathcal{L}^{\prime} & \frac{1}{2} & \mathcal{J}^{\prime} \\
R & 1 & Z
\end{array}\right\}\left\{\begin{array}{ccc}
j & j^{\prime} & Z \\
\mathcal{J}^{\prime} & \mathcal{J} & J
\end{array}\right\}\left\{\begin{array}{ccc}
Z & 1 & Y \\
K_{1} & V & 1
\end{array}\right\}\left\{\begin{array}{ccc}
Z & 1 & Y \\
K_{2} & R & 1
\end{array}\right\} \\
& \times \sum_{K_{3}=0}^{K 2}\left[\left(\begin{array}{c}
2 K_{2}+1 \\
2 K_{3}
\end{array}\right)\right]^{\frac{1}{2}} \widehat{K_{2}-K_{3}} \sum_{X} \hat{X}^{2}\left(X 0 K_{3} 0 \mid Y 0\right)\left(X 0 K_{2}-K_{3} 0 \mid R 0\right)\left\{\begin{array}{ccc}
Y & X & K_{3} \\
K_{2}-K_{3} & K_{2} & R
\end{array}\right\} \\
& \times \int \mathrm{d} \xi_{1} \mathrm{~d} \xi_{2} \xi_{1}^{2} \xi_{2}^{2} R_{n l}\left(\xi_{1}, b\right) R_{\mathcal{N} \mathcal{L}}\left(\xi_{2}, b\right) R_{n^{\prime} l^{\prime}}\left(\xi_{1}, b\right) R_{\mathcal{N}^{\prime} \mathcal{L}^{\prime}}\left(\xi_{2}, b\right) \\
& \times\left(\sqrt{\frac{1}{2}} \xi_{1}\right)^{K_{3}}\left(\sqrt{\frac{3}{2}} \xi_{2}\right)^{K_{2}-K_{3}} f_{K_{1}}\left(\sqrt{2} \xi_{1} ; \Lambda\right) f_{K_{2}, X}\left(\sqrt{\frac{1}{2}} \xi_{1}, \sqrt{\frac{3}{2}} \xi_{2} ; \Lambda\right),
\end{aligned}
$$


with the function $f_{K_{1}}(r ; \Lambda)$ given by (39), the function $f_{0, X}\left(r_{1}, r_{2} ; \Lambda\right)$ given by (34) and the function $f_{2, X}\left(r_{1}, r_{2} ; \Lambda\right)$ given by (35).

Finally, the $W_{1}$ part of two-pion exchange $c_{4}$ term is given by [11]

$$
W_{1}^{2 \pi_{-} c 4}=c_{4} \frac{1}{(2 \pi)^{6}} \frac{1}{F_{\pi}^{2}} \frac{g_{\mathrm{A}}^{2}}{4 F_{\pi}^{2}} \vec{\tau}_{1} \cdot\left(\vec{\tau}_{2} \times \vec{\tau}_{3}\right) F\left(\vec{Q}^{2} ; \Lambda\right) \frac{1}{\vec{Q}^{2}+M_{\pi}^{2}} \vec{\sigma}_{2} \cdot \vec{Q} \vec{\sigma}_{3} \cdot \vec{Q}^{\prime} \vec{\sigma}_{1} \cdot\left(\vec{Q} \times \vec{Q}^{\prime}\right) \frac{1}{\vec{Q}^{\prime 2}+M_{\pi}^{2}} F\left(\vec{Q}^{\prime 2} ; \Lambda\right),
$$

and for its matrix element we find

$$
\begin{aligned}
& \left\langle(n l s j t, \mathcal{N} \mathcal{L} \mathcal{J}) J T\left|W_{1}^{2 \pi_{-} \mathrm{c} 4, \mathrm{Q}}\right|\left(n^{\prime} l^{\prime} s^{\prime} j^{\prime} t^{\prime}, \mathcal{N}^{\prime} \mathcal{L}^{\prime} \mathcal{J}^{\prime}\right) J T\right\rangle
\end{aligned}
$$

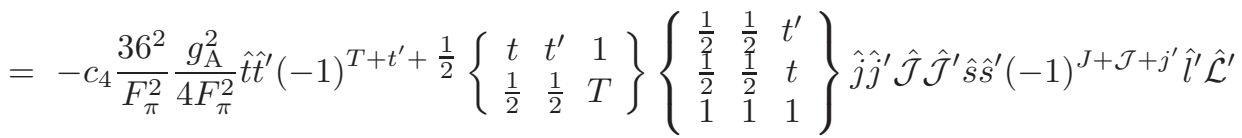

$$
\begin{aligned}
& \times \sum_{K_{1} K_{2}}(-1)^{\left(K_{1}+K_{2}\right) / 2} \hat{K}_{1} \hat{K}_{2}\left(1010 \mid K_{1} 0\right)\left(1010 \mid K_{2} 0\right) \\
& \times \sum_{V R}(-1)^{V+R} \hat{V} \hat{R}\left(V 0 l^{\prime} 0 \mid l 0\right)\left(R 0 \mathcal{L}^{\prime} 0 \mid \mathcal{L} 0\right) \sum_{Y} \hat{Y}\left(Y 0 K_{1} 0 \mid V 0\right) \\
& \times \sum_{Z} \hat{Z}^{2}(-1)^{Z}\left\{\begin{array}{ccc}
\mathcal{L} & \frac{1}{2} & \mathcal{J} \\
\mathcal{L}^{\prime} & \frac{1}{2} & \mathcal{J}^{\prime} \\
R & 1 & Z
\end{array}\right\}\left\{\begin{array}{ccc}
j & j^{\prime} & Z \\
\mathcal{J}^{\prime} & \mathcal{J} & J
\end{array}\right\}\left\{\begin{array}{ccc}
Z & 1 & Y \\
K_{2} & R & 1
\end{array}\right\} \\
& \times \sum_{K_{4}} \hat{K}_{4}^{2}\left\{\begin{array}{ccc}
l & s & j \\
l^{\prime} & s^{\prime} & j^{\prime} \\
V & K_{4} & Z
\end{array}\right\}\left\{\begin{array}{ccc}
\frac{1}{2} & \frac{1}{2} & s \\
\frac{1}{2} & \frac{1}{2} & s^{\prime} \\
1 & 1 & K_{4}
\end{array}\right\}\left\{\begin{array}{ccc}
Z & 1 & Y \\
K_{1} & V & K_{4}
\end{array}\right\}\left\{\begin{array}{ccc}
1 & 1 & 1 \\
1 & K_{4} & K_{1}
\end{array}\right\} \\
& \times \sum_{K_{3}=0}^{K 2}\left[\left(\begin{array}{c}
2 K_{2}+1 \\
2 K_{3}
\end{array}\right)\right]^{\frac{1}{2}} \widehat{K_{2}-K_{3}} \sum_{X} \hat{X}^{2}\left(X 0 K_{3} 0 \mid Y 0\right)\left(X 0 K_{2}-K_{3} 0 \mid R 0\right)\left\{\begin{array}{ccc}
Y & X & K_{3} \\
K_{2}-K_{3} & K_{2} & R
\end{array}\right\} \\
& \times \int \mathrm{d} \xi_{1} \mathrm{~d} \xi_{2} \xi_{1}^{2} \xi_{2}^{2} R_{n l}\left(\xi_{1}, b\right) R_{\mathcal{N} \mathcal{L}}\left(\xi_{2}, b\right) R_{n^{\prime} l^{\prime}}\left(\xi_{1}, b\right) R_{\mathcal{N}^{\prime} \mathcal{L}^{\prime}}\left(\xi_{2}, b\right) \\
& \times\left(\sqrt{\frac{1}{2}} \xi_{1}\right)^{K_{3}}\left(\sqrt{\frac{3}{2}} \xi_{2}\right)^{K_{2}-K_{3}} f_{K_{1}}\left(\sqrt{2} \xi_{1} ; \Lambda\right) f_{K_{2}, X}\left(\sqrt{\frac{1}{2}} \xi_{1}, \sqrt{\frac{3}{2}} \xi_{2} ; \Lambda\right) .
\end{aligned}
$$

The same functions (34), (35) and (39) that were introduced in the $c_{3}$ term enter the $c_{4}$ term as well.

We note that the local two-pion-exchange terms appear also in the Tucson-Melbourne NNN interaction [20]. The analogous terms to $c_{1}, c_{3}$ and $c_{4}$ are present in particular in the $\mathrm{TM}^{\prime}$ interaction [21, 22]. The $\mathrm{TM}^{\prime}$ parameters are denoted by $a^{\prime}, b$ and $d$ with the relation to the above $c_{1}, c_{3}$ and $c_{4}$ given by

$$
\begin{aligned}
a^{\prime} & =\frac{4 M_{\pi}^{2}}{F_{\pi}^{2}} c_{1}, \\
b & =\frac{2}{F_{\pi}^{2}} c_{3}, \\
d & =\frac{-1}{F_{\pi}^{2}} c_{4} .
\end{aligned}
$$

Further, the regulator function $F\left(q^{2} ; \Lambda\right)$ is chosen in the form

$$
F^{T M}\left(q^{2} ; \Lambda\right)=\frac{\Lambda^{2}-M_{\pi}^{2}}{\Lambda^{2}+q^{2}} .
$$

This choice allows to evaluate integrals that define the functions $f_{K}$ analytically. The analytic expressions can be found, e.g. in Ref. [29]. In that paper, the following function is introduced:

$$
Z_{1}(r ; \Lambda)=\frac{1}{2 \pi^{2}} \int \mathrm{d} q q^{2} j_{0}(q r) \frac{F\left(q^{2} ; \Lambda\right)}{q^{2}+M_{\pi}^{2}} .
$$

Using the properties of spherical Bessel functions, we can easily find relations between derivatives of $Z_{1}$ and our $f_{K}$ 
functions:

$$
\begin{aligned}
& f_{0}(r, \Lambda)=-\left(Z_{1}^{\prime \prime}(r ; \Lambda)+\frac{2}{r} Z_{1}^{\prime}(r ; \Lambda)\right), \\
& f_{1}(r, \Lambda)=-Z_{1}^{\prime}(r ; \Lambda) \\
& f_{2}(r, \Lambda)=Z_{1}^{\prime \prime}(r ; \Lambda)-\frac{1}{r} Z_{1}^{\prime}(r ; \Lambda) .
\end{aligned}
$$

For completeness, we note that a still different notation was used in Ref. [10], where functions $I_{k, l}(r ; \Lambda)$ were introduced. They are related to the $Z_{0}(r, \Lambda)$ that we introduced in Eq. (13) and to the above $Z_{1}$ function (54) is as follows:

$$
\begin{aligned}
& Z_{0}(r, \Lambda)=I_{0,0}(r ; \Lambda), \\
& Z_{1}(r, \Lambda)=I_{2,0}(r, \Lambda) .
\end{aligned}
$$

In Ref. [36], the Tucson-Melbourne NNN interaction matrix elements in the HO basis were calculated using a different algorithm than the one used in this paper. That algorithm relied on a completeness relation and transformations of HO states with the help of HO brackets. Even though the algorithm of Ref. [36] required calculation of onedimensional radial integrals, while the the present algorithm requires evaluation of two-dimensional radial integrals, the present algorithm is substantially more efficient.

\section{CONVERGENCE TEST FOR ${ }^{3} \mathrm{H}$ AND ${ }^{4} \mathrm{HE}$}

In this section, we apply the matrix elements of the $\mathrm{N}^{2} \mathrm{LO} \chi \mathrm{EFT}$ NNN interaction obtained in this paper to the NCSM calculation of ${ }^{3} \mathrm{H}$ ad ${ }^{4} \mathrm{He}$ ground state properties. As a test of correctness of the computer code, we verified that the new more efficient algorithm reproduces the results obtained using the algorithm of Ref. [36] for the twopion-exchange term matrix elements. For the contact terms, we verified that in the limit of $\Lambda \rightarrow \infty$, the matrix elements (15) and (14) lead to the same result and the same is true for matrix elements (38) and (30). In addition, we benchmarked the computer code for evaluation of (14) and (30) with the computer code written by A. Nogga [37]. Finally, we tested numerically that the use of (33) results in the same matrix element as the use of (38) in the threenucleon antisymmetrized basis $|N i J T\rangle$ introduced in Eq. (8). The same checks were also performed for the alternative version of the one-pion-exchange plus contact term $(D$-term) given in Eq. (29). That is, we verified numerically that the matrix element (40) leads to the same result as (30) in the limit of $\Lambda \rightarrow \infty$ and the use of (40) results in the same matrix element in the three-nucleon antisymmetrized basis $|N i J T\rangle$ as the use of (33) multiplied by $(-1)^{t+t^{\prime}+s+s^{\prime}}$.

We use the $\mathrm{N}^{3} \mathrm{LO} \mathrm{NN}$ interaction of Ref. [13]. We adopt the $c_{1}, c_{3}$ and $c_{4}$ LECs values as well as the value of $\Lambda$ from the $\mathrm{N}^{3} \mathrm{LO} \mathrm{NN}$ interaction of Ref. [13] for our local chiral EFT $\mathrm{N}^{2} \mathrm{LO} N \mathrm{NN}$ interaction. The regulator function was chosen in a form consistent with that used in Refs. [11] and [13]: $F\left(q^{2} ; \Lambda\right)=\exp \left(-q^{4} / \Lambda^{4}\right)$ (11). Values of the $c_{D}$ and $c_{E}$ LECs are constrained by a fit to the $A=3$ system binding energy 14, 18]. Obviously, additional constraints are needed to uniquely determine values of $c_{D}$ and $c_{E}$, see Refs. [11, 14, 18, 38] for discussions of different possibilities. Here we are interested only in convergence properties of our calculations. Therefore, we simply select a reasonable value, e.g. $c_{D}=1$, and follow Ref. [14] and adopt $c_{E}$ value as an average of fits to ${ }^{3} \mathrm{H}$ and ${ }^{3} \mathrm{He}$ binding energies. In Table I. we summarize the NNN interaction parameters used in calculations described in this section. We note that ${ }^{4} \mathrm{He}$ results obtained with the identical Hamiltonian but with $c_{D}=-1$ and $c_{E}=-0.331$ are presented in Ref. [17].

TABLE I: NNN interaction parameters used in the present calculations. The regulator function was chosen in the form $F\left(q^{2} ; \Lambda\right)=\exp \left(-q^{4} / \Lambda^{4}\right)$.

\begin{tabular}{cccccccccc}
\hline \hline$c_{1}\left[\mathrm{GeV}^{-} 1\right]$ & $c_{3}\left[\mathrm{GeV}^{-} 1\right]$ & $c_{4}\left[\mathrm{GeV}^{-} 1\right]$ & $c_{D}$ & $c_{E}$ & $\Lambda[\mathrm{MeV}]$ & $\Lambda_{\chi}[\mathrm{MeV}]$ & $M_{\pi}[\mathrm{MeV}]$ & $g_{A}$ & $F_{\pi}[M e V]$ \\
\hline-0.81 & -3.2 & 5.4 & 1.0 & -0.029 & 500 & 700 & 138 & 1.29 & 92.4 \\
\hline \hline
\end{tabular}

We use the Jacobi coordinate HO basis antisymmentrized according to the method described in Ref. 35. In Figs. 6 and 7, we show the convergence of the ${ }^{3} \mathrm{H}$ ground-state energy and point-proton rms radius, respectively, with the size of the basis. Thin lines correspond to results obtained with the NN interaction only. Thick lines correspond to calculations that also include the NNN interaction. The full lines correspond to calculations with two-body effective interaction derived from the chiral EFT $\mathrm{N}^{3} \mathrm{LO} \mathrm{NN}$ interaction. The dashed lines correspond to calculations with the bare chiral EFT $\mathrm{N}^{3} \mathrm{LO}$ NN interaction. The bare NNN interaction is added to either the bare NN or to the effective $\mathrm{NN}$ interaction in calculations depicted by thick lines. We observe that the convergence is faster when the two-body effective interaction is used. However, starting at about $N_{\max }=24$ the convergence is reached also in calculations 
with the bare NN interaction. The rate of convergence also depends on the choice of the HO frequency. In general, it is always advantageous to use the effective interaction in order to improve the convergence rate. The ${ }^{3} \mathrm{H}$ ground-state energy and point-proton radius results are summarized in Table II The contributions of different NNN terms to the ${ }^{3} \mathrm{H}$ ground-state energy are presented in Table III In addition to results obtained using the $c_{D}=1$, we also show in Table III results obtained using $c_{D}=-1$ and a corresponding $c_{E}$ constrained by the avarage of the ${ }^{3} \mathrm{H}$ and ${ }^{3} \mathrm{He}$ binding energy fit. For completeness, we show results obtained by the two alternative one-pion-exchange plus contact terms (28) and (29). In all cases, the contact $E$-term gives a positive contribution. The contribution from the $D$-term changes sign depending on the choice of $c_{D}$. Still, the two-pion exchange $c$-terms dominate the NNN expectation value.

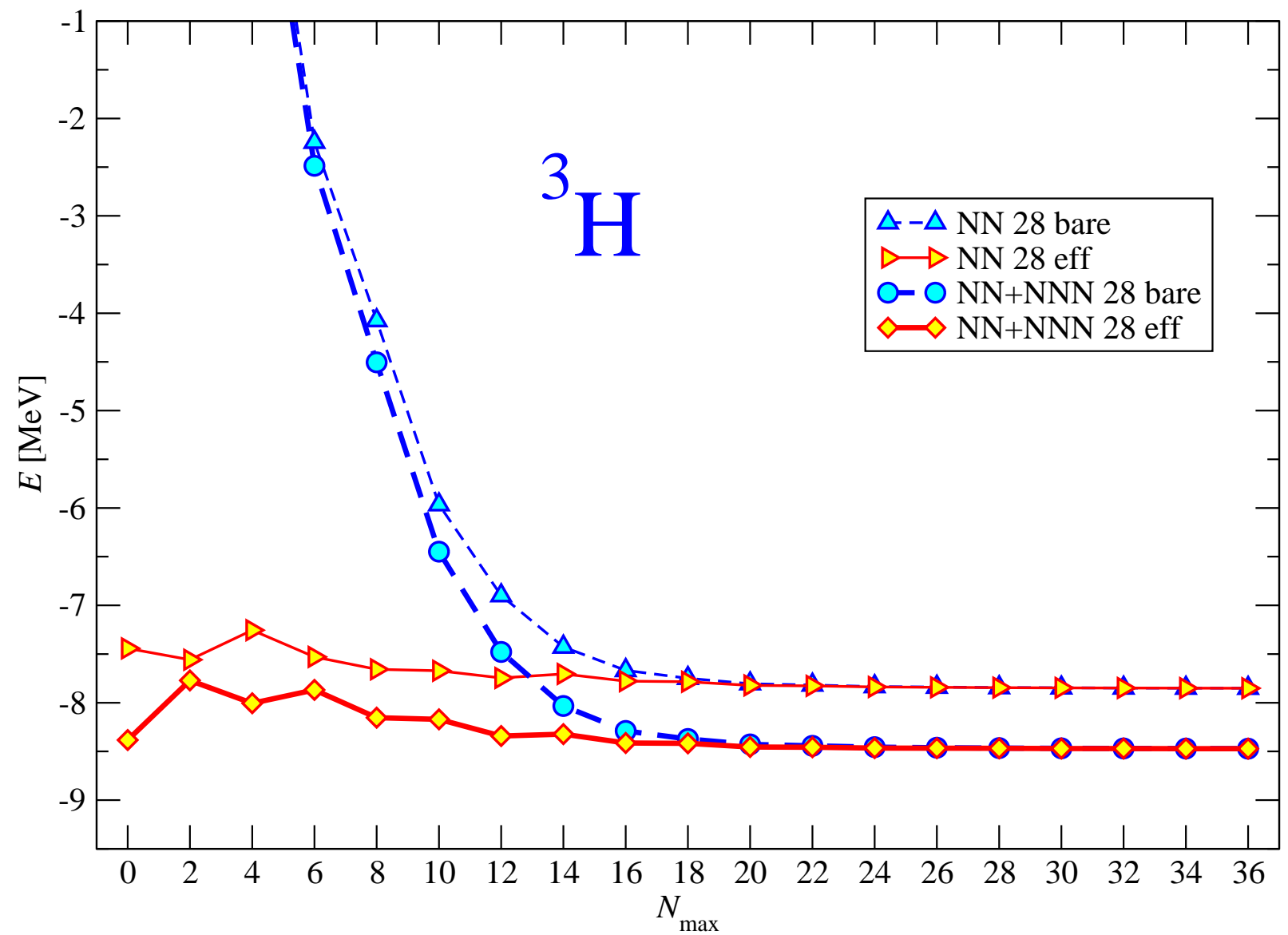

FIG. 6: (Color online) ${ }^{3} \mathrm{H}$ ground-state energy dependence on the size of the basis. The HO frequency of $\hbar \Omega=28 \mathrm{MeV}$ was employed. Results with (thick lines) and without (thin lines) the NNN interaction are shown. The full lines correspond to calculations with two-body effective interaction derived from the chiral NN interaction, the dashed lines to calculations with the bare chiral NN interaction. For further details see the text.

In Figs. 8 and 9 we show convergence of the ${ }^{4} \mathrm{He}$ ground-state energy and point-proton rms radius, respectively. The NCSM calculations are perforemed in basis spaces up to $N_{\max }=20$. Thin lines correspond to results obtained with the NN interaction only, while thick lines correspond to calculations that also include the NNN interaction. The dashed lines correspond to results obtained with bare interactions. The full lines correspond to results obtained using three-body effective interaction (the NCSM three-body cluster approximation). It is apparent that the use of the three-body effective interaction improves the convergence rate dramatically. We can see that at about $N_{\max }=18$ the bare interaction calculation reaches convergence as well. It should be noted, however, that $p$-shell calculations with the NNN interactions are presently feasible in model spaces up to $N_{\max }=6$ or $N_{\max }=8$. The use of the three-body effective interaction is then essential in the $p$-shell calculations.

We note that NCSM calculations in the three-body cluster approximation are rather involved. The ${ }^{4} \mathrm{He}$ NCSM calculation with the three-body effective interaction proceeds in three steps. First, we diagonalize the Hamiltonian with and without the NNN interaction in a three-nucleon basis for all relevant three-body channels. In the second step, we use the three-body solutions from the first step to derive three-body effective interactions with and without 


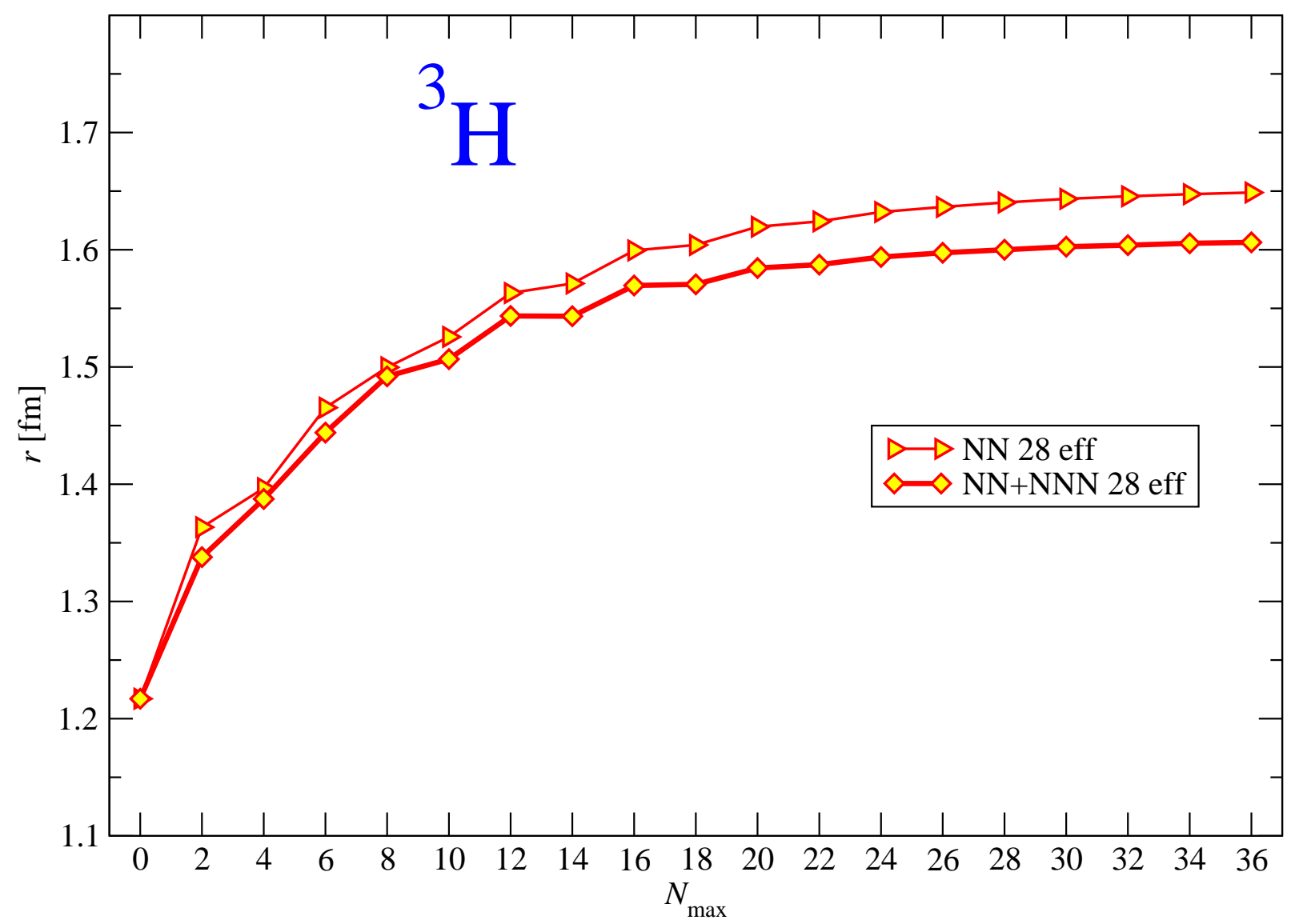

FIG. 7: (Color online) ${ }^{3} \mathrm{H}$ point-proton rms radius dependence on the size of the basis. The HO frequency of $\hbar \Omega=28 \mathrm{MeV}$ was employed. Results with (thick line) and without (thin line) the NNN interaction are shown. The two-body effective interaction derived from the chiral NN interaction was used in the calculation. For further details see the text.

TABLE II: Ground-state energy and point-proton rms radius of ${ }^{3} \mathrm{H}$ and ${ }^{4} \mathrm{He}$ calculated using the chiral $\mathrm{N}^{3} \mathrm{LO}$ NN potential 13] with and without the local chiral $\mathrm{N}^{2} \mathrm{LO}$ NNN interaction. The LECs values and other parameters are given in Table I] The calculations were performed within the ab initio NCSM.

\begin{tabular}{|c|c|c|c|}
\hline \multicolumn{4}{|c|}{${ }^{3} \mathrm{H}$} \\
\hline & NN & $\mathrm{NN}+\mathrm{NNN}$ & Expt. \\
\hline$E_{\mathrm{gs}}[\mathrm{MeV}]$ & $-7.852(5)$ & $-8.473(5)$ & -8.482 \\
\hline$r_{p}[\mathrm{fm}]$ & $1.650(5)$ & $1.608(5)$ & \\
\hline \multicolumn{4}{|c|}{${ }^{4} \mathrm{He}$} \\
\hline & NN & $\mathrm{NN}+\mathrm{NNN}$ & Expt. \\
\hline$E_{\mathrm{gs}}[\mathrm{MeV}]$ & $-25.39(1)$ & $-28.34(2)$ & -28.296 \\
\hline$r_{p}[\mathrm{fm}]$ & $1.515(2)$ & $1.475(2)$ & $1.455(7)$ \\
\hline
\end{tabular}

TABLE III: Contributions of different NNN terms to the ${ }^{3} \mathrm{H}$ ground-state energy. The $c_{D}$ and $c_{E}$ LECs are explicitly shown. Other parameters are given in Table I All energies are given in MeV. The two alternative one-pion-exchange plus contact terms (28) and (29) are considered.

\begin{tabular}{|c|c|c|c|c|c|}
\hline \multicolumn{6}{|c|}{${ }^{3} \mathrm{H}$} \\
\hline$c_{D}$ & $c_{E}$ & $E_{\mathrm{gs}}$ & $c$ terms & $D$ term & $E$ term \\
\hline 1.0 (Eq. (28)) & -0.029 & -8.473 & -1.01 & 0.13 & 0.03 \\
\hline-1.0 (Eq. (28) & -0.331 & -8.474 & -1.07 & -0.16 & 0.32 \\
\hline 1.0 (Eq. (29) & -0.159 & -8.471 & -0.99 & 0.005 & 0.14 \\
\hline-1.0 (Eq. (29) & -0.213 & -8.474 & -1.10 & -0.05 & 0.21 \\
\hline
\end{tabular}




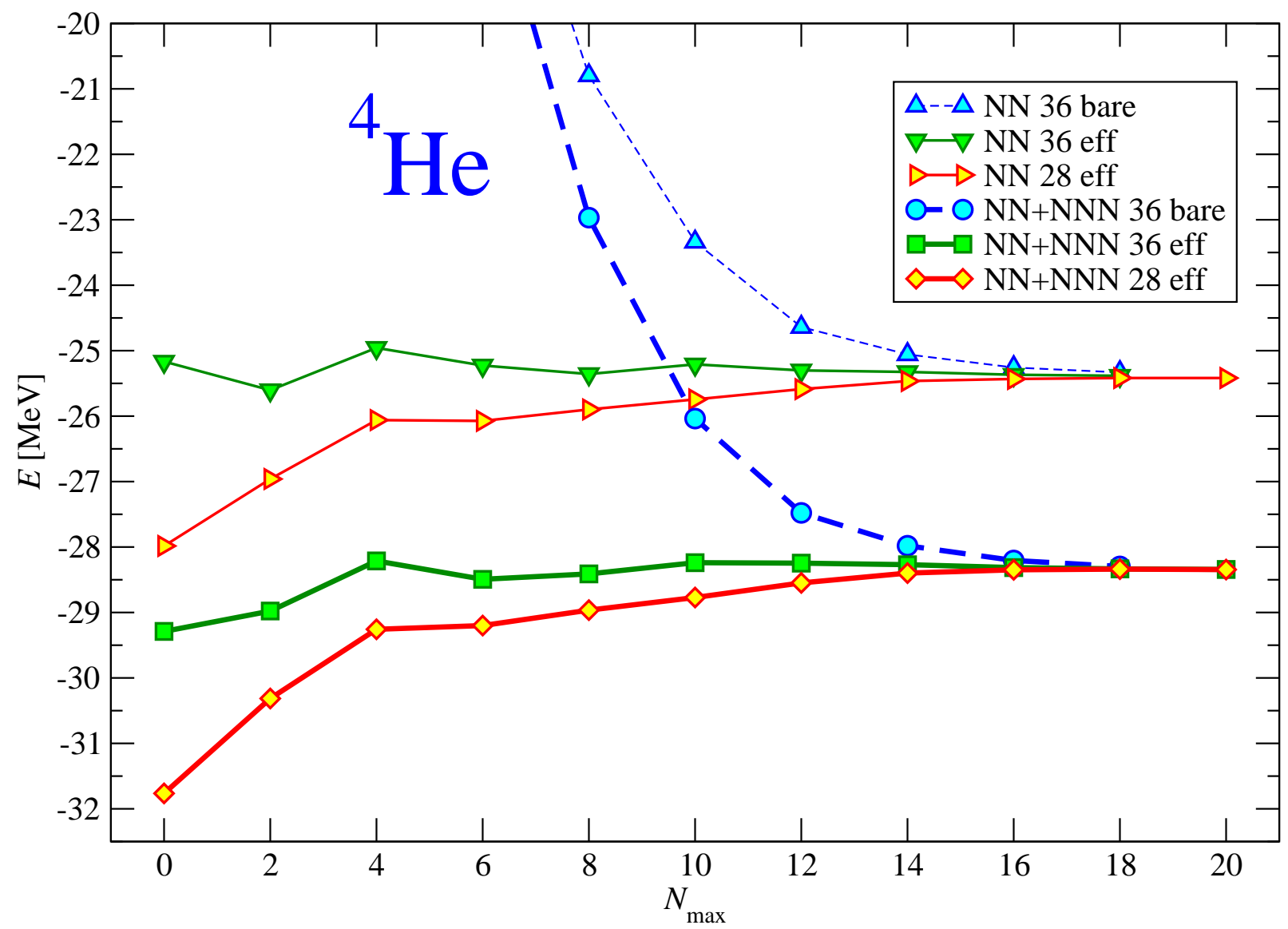

FIG. 8: (Color online) ${ }^{4} \mathrm{He}$ ground-state energy dependence on the size of the basis. The HO frequencies of $\hbar \Omega=28$ and $36 \mathrm{MeV}$ was employed. Results with (thick lines) and without (thin lines) the NNN interaction are shown. The full lines correspond to calculations with three-body effective interaction, the dashed lines to calculations with the bare interaction. For further details see the text.

the NNN interaction. By subtracting the two effective interactions we isolate the NN and NNN contributions. This is needed due to a different scaling with particle number of the two- and the three-body interactions. The ${ }^{4} \mathrm{He}$ efffective interaction is then obtained by adding the two contributions with the appropriate scaling factors [16]. In the third step, we diagonalize the resulting Hamiltonian in the antisymmetrized four-nucleon Jacobi-coordinate HO basis to obtain the ${ }^{4} \mathrm{He} J^{\pi} T=0^{+} 0$ ground state. Obviously, in calculations without the NNN interaction, the above three steps are simplified as no NNN contribution needs to be isolated. In addition, in the case of no NNN interaction, we may use just the two-body effective interaction (two-body cluster approximation), which is much simpler. The convergence is slower, however, see discussion in Ref. [39]. We also note that ${ }^{4} \mathrm{He}$ properties with the chiral $\mathrm{N}^{3} \mathrm{LO}$ NN interaction that we employ here were calculated using two-body cluster approximation in Ref. [40] and present results are in agreement with results found there.

Our ${ }^{4} \mathrm{He}$ results are summarized in Table [I] We note that the present NCSM ${ }^{3} \mathrm{H}$ and ${ }^{4} \mathrm{He}$ results obtained with the chiral $\mathrm{N}^{3} \mathrm{LO} \mathrm{NN}$ interaction are in a perfect agreement with results obtained using the variational calculations in the hyperspherical harmonics basis as well as with the Faddeev-Yakubovsky calculations published in Ref. [41]. A satisfying feature of the present NCSM calculation is the fact that the rate of convergence is not affected in any significant way by inclusion of the NNN interaction.

\section{CONCLUSIONS}

In this paper, we regulated the $\mathrm{NNN}$ interaction derived within the chiral effective field theory at the $\mathrm{N}^{2} \mathrm{LO}$ with a function depending on the magnitude of the momentum transfer. The regulated NNN interaction is local in the coordinate space. This is advantages for some many-body techniques. In addition, it was found that this interaction performs sligthtly better in mid-p-shell nuclei than its nonlocal counterpart [14, 37]. We calculated matrix elements 


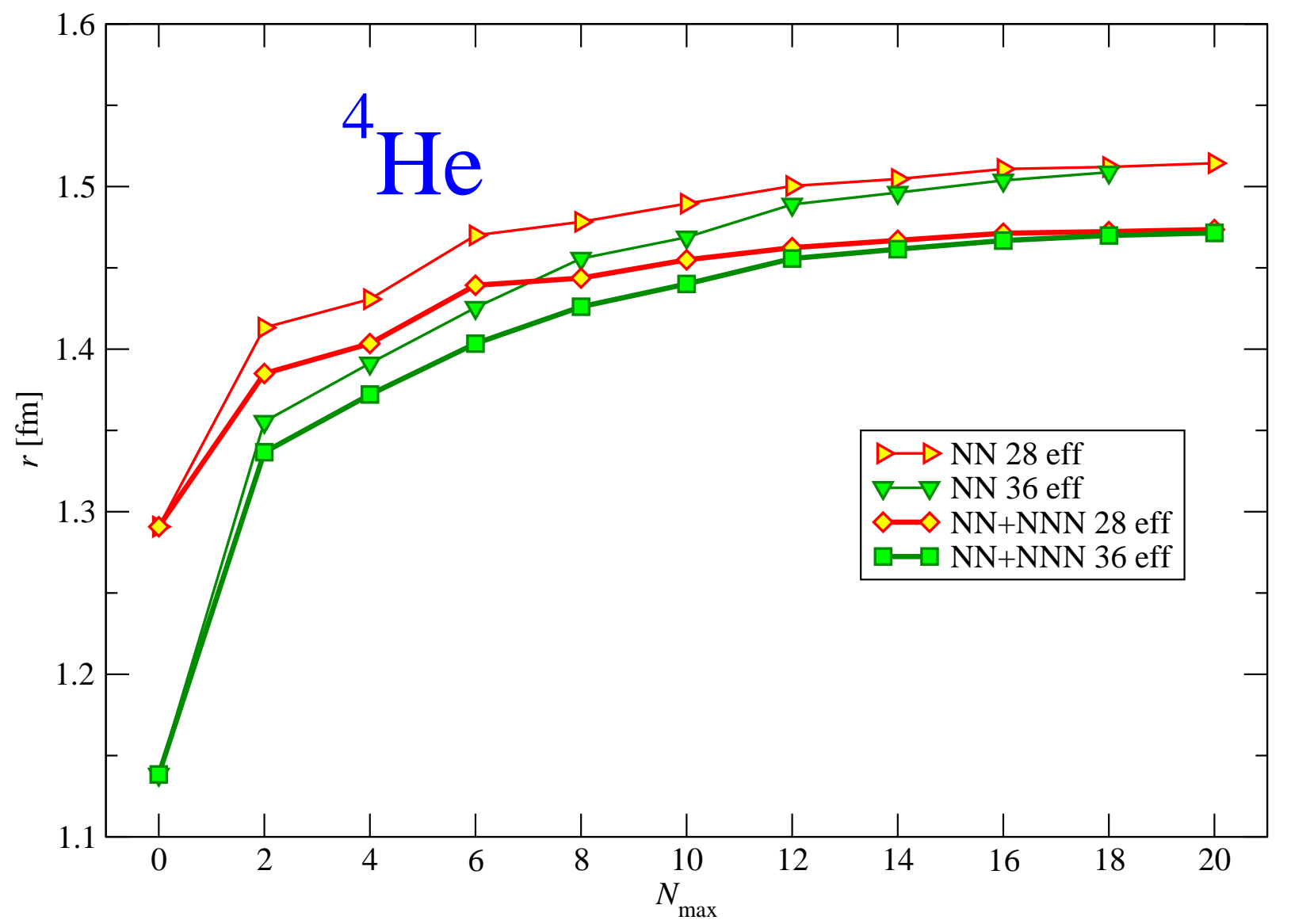

FIG. 9: (Color online) ${ }^{4}$ He point-proton rms radius dependence on the size of the basis. The HO frequencies of $\hbar \Omega=28$ and 36 $\mathrm{MeV}$ was employed. Results with (thick line) and without (thin line) the NNN interaction are shown. The three-body effective interaction was used in the calculation. For further details see the text.

of the local chiral NNN interaction in the three-nucleon $\mathrm{HO}$ basis and performed calculations for ${ }^{3} \mathrm{H}$ and ${ }^{4} \mathrm{He}$ within the $a b$ initio NCSM. We demonstrated that a very good convergence of the ground-state properties of these nuclei remains unchanged when the NNN interaction is added to the Hamiltonian. Expressions for the local $\chi \mathrm{EFT}$ NNN interaction matrix elements derived in this paper may by used after some modifications with other bases, e.g. with the hyperspherical harmonics basis.

\section{Acknowledgments}

I would like to thank U. van Kolck, E. Epelbaum and J. Adam, Jr. for useful comments and A. Nogga for code benchmarking. This work was performed under the auspices of the U. S. Department of Energy by the University of California, Lawrence Livermore National Laboratory under contract No. W-7405-Eng-48. Support from the LDRD contract No. 04-ERD-058 and from U.S. DOE/SC/NP (Work Proposal Number SCW0498) is acknowledged. This work was also supported in part by the Department of Energy under Grant DE-FC02-07ER41457.

[1] S. Weinberg, Physica 96A, 327 (1979); Phys. Lett. B 251, 288 (1990); Nucl. Phys. B363, 3 (1991); J. Gasser et al., Ann. of Phys. 158, 142 (1984). Nucl. Phys. B250, 465 (1985).

[2] V. Bernard, N. Kaiser, and Ulf-G. Meißner, Int. J. Mod. Phys. E4, 193 (1995).

[3] C. Ordonez, L. Ray, and U. van Kolck, Phys. Rev. Lett. 72, 1982 (1994). Phys. Rev. C 53, 2086 (1996).

[4] U. van Kolck, Prog. Part. Nucl. Phys. 43, 337 (1999). 
[5] P. F. Bedaque and U. van Kolck, Ann. Rev. Nucl. Part. Sci. 52, 339 (2002); E. Epelbaum, Prog. Part. Nucl. Phys. 57, 654 (2006).

[6] S. R. Beane, P. F. Bedaque, K. Orginos, and M. J. Savage, Phys. Rev. Lett. 97, 012001 (2006).

[7] S. R. Beane, P. F. Bedaque, M. J. Savage and U. van Kolck, Nucl. Phys. A700, 377 (2002); A. Nogga, R. G. Timmermans, and U. van Kolck, Phys. Rev. C 72, 054006 (2005).

[8] M. C. Birse, Phys. Rev. C 74, 014003 (2006).

[9] E. Epelbaum and U.-G. Meissner, nucl-th/0609037.

[10] U. van Kolck, Phys. Rev. C 49, 2932 (1994).

[11] E. Epelbaum, A. Nogga, W. Glöckle, H. Kamada, Ulf-G. Meissner and H. Witala, Phys. Rev. C 66, 064001 (2002).

[12] E. Epelbaum, Phys. Lett. B 639, 456 (2006).

[13] D. R. Entem and R. Machleidt, Phys. Rev. C 68, 041001(R) (2003).

[14] P. Navrátil and V. G. Gueorguiev and J. P. Vary, W. E. Ormand and A. Nogga, Phys. Rev. Lett. 99, 042501 (2007); nucl-th/0701038

[15] P. Navrátil, J. P. Vary and B. R. Barrett, Phys. Rev. Lett. 84, 5728 (2000); Phys. Rev. C 62, 054311 (2000).

[16] P. Navrátil and W. E. Ormand, Phys. Rev. C 68, 034305 (2003).

[17] S. Quaglioni and P. Navrátil, Phys. Lett. B (2007), doi:10.1016/j.physletb.2007.06.082; arXiv:0704.1336

[18] A. Nogga, P. Navrátil, B. R. Barrett and J. P. Vary Phys. Rev. C 73, 064002 (2006).

[19] B. S. Pudliner, V. R. Pandharipande, J. Carlson, and R. B. Wiringa, Phys. Rev. Lett. 74, 4396 (1995).

[20] S. A. Coon, M. D. Scadron, P. C. McNamee, B. R. Barrett, D. W. E. Blatt and B. H. J. McKellar, Nucl. Phys. A 317, 242 (1979).

[21] J. L. Friar, D. Hüber, and U. van Kolck, Phys. Rev. C 59, 53 (1999).

[22] S. A. Coon and H. K. Han, Few-Body Systems 30, 131 (2001).

[23] S. C. Pieper, Nucl. Phys. A571, 516 (2005).

[24] S. C. Pieper, R. B. Wiringa and J. Carlson, Phys. Rev. C 70, 054325 (2004).

[25] R. B. Wiringa, V. G. J. Stoks, and R. Schiavilla, Phys. Rev. C 51, 38 (1995).

[26] S. C. Pieper, V. R. Pandharipande, R. B. Wiringa, and J. Carlson, Phys. Rev. C 64, 014001 (2001).

[27] S. C. Pieper, K. Varga and R. B. Wiringa, Phys. Rev. C 66, 044310 (2002); R. B. Wiringa and S. C. Pieper, Phys. Rev. Lett. 89, 182501 (2002).

[28] S. A. Coon and W. Glöckle, Phys. Rev. C 23 1790, (1981).

[29] J. L. Friar, B. F. Gibson, G. L. Payne, and S. A. Coon, Few-Body Systems 5, 13 (1988).

[30] S. A. Coon and M. T. Peña, Phys. Rev. C 48, 2559 (1993).

[31] D. Hüber, H. Witala, A. Nogga, W. Glöckle, and H. Kamada, Few-Body Systems 22, 107 (1997).

[32] D. Hüber, J. L. Friar, A. Nogga, H. Witala, and U. van Kolck, Few-Body Systems 30, 95 (2001).

[33] N. Barnea, V. D. Efros, W. Leidemann, and G. Orlandini, Few-Body Systems 35, 155 (2004).

[34] J. Adam, Jr., M. T. Peña, and A. Stadler, Phys. Rev. C 69, 034008 (2004).

[35] P. Navrátil, G. P. Kamuntavičius, and B. R. Barrett, Phys. Rev. C 61, 044001 (2000).

[36] D. C. J. Marsden, P. Navrátil, S. A. Coon and B. R. Barrett, Phys. Rev. C 66, 044007 (2002).

[37] A. Nogga, private communication.

[38] C. Hanhart, U. van Kolck, and G. A. Miller, Phys. Rev. Lett. 85, 2905 (2000).

[39] P. Navrátil and W. E. Ormand, Phys. Rev. Lett. 88 (2002) 152502.

[40] P. Navrátil and E. Caurier, Phys. Rev. C 69 (2004) 014311.

[41] M. Viviani, L. E. Marcucci, S. Rosati, A. Kievsky and L. Girlanda, Few-Body Systems 39, 159 (2006). 